\title{
Comparison of psychometric properties between recall methods of interview-based physical activity questionnaires: a systematic review
}

\author{
Kenji Doma ${ }^{1 *}$ (D) Renée Speyer ${ }^{2,3,4}$, Lauren Alese Parsons ${ }^{3}$ and Reinie Cordier ${ }^{3}$
}

\begin{abstract}
Background: This systematic review examined the methodological quality of studies and assessed the psychometric qualities of interview-administered Past-week and Usual-week Physical Activity Questionnaires (PAQs). Pubmed and Embase were used to retrieve data sources.

Methods: The studies were selected using the following eligibility criteria: 1) psychometric properties of PAQs were assessed in adults; 2) the PAQs either consisted of recall periods of usual 7-days (Usual-week PAQs) within the past 12 months or during the past 7-days (Past-week PAQs); and 3) PAQs were interview-administered. The COSMIN taxonomy was utilised to critically appraise study quality and a previously established psychometric criteria employed to evaluate the overall psychometric qualities.

Results: Following screening, 42 studies were examined to determine the psychometric properties of 20 PAQs, with the majority of studies demonstrating good to excellent ratings for methodological quality. For convergent validity (i.e., the relationship between PAQs and other measures), similar overall associations were found between Past-week PAQs and Usual-week PAQs. However, PAQs were more strongly associated with direct measures of physical activity (e.g., accelerometer) than indirect measures of physical activity (i.e., physical fitness), irrespective of recall methods. Very few psychometric properties were examined for each PAQ, with the majority exhibiting poor ratings in psychometric quality. Only a few interview-administered PAQs exhibited positive ratings for a single psychometric property, although the other properties were either rated as poor or questionable, demonstrating the limitations of current PAQs.
\end{abstract}

Conclusion: Accordingly, further research is necessary to explore a greater number of psychometric properties, or to develop new PAQs by addressing the psychometric limitations identified in the current review.

Keywords: Recall methods, Validity, Reliability, Direct measures, Indirect measures, COSMIN

\section{Background}

The prevalence and severity of obesity is continually increasing in most of the Western world, developing into epidemic proportions worldwide [1]. Lack of physical activity reduces physical fitness, and is a major contributor to this global health crisis and is associated with development of chronic diseases and cancer, leading to increased

\footnotetext{
* Correspondence: kenji.doma@jcu.edu.au

${ }^{1}$ College of Healthcare Sciences, James Cook University, Townsville,

Queensland, Australia

Full list of author information is available at the end of the article
}

mortality [2]. In contrast, participation in physical activity has been associated with improved health outcomes, lower incidences of health problems and reduced mortality rates [3-5]. International guidelines developed by the World Health Organisation (WHO) recommend that children and adults to engage in $60 \mathrm{~min}$ of moderateto-vigorous physical activity each day $[6,7]$. To assess whether physical activity is being performed at these recommended levels, adequate monitoring of patient's lifestyles and behaviours is needed [8]. This enables health

(c) The Author(s). 2019 Open Access This article is distributed under the terms of the Creative Commons Attribution 4.0 International License (http://creativecommons.org/licenses/by/4.0/), which permits unrestricted use, distribution, and reproduction in any medium, provided you give appropriate credit to the original author(s) and the source, provide a link to the Creative Commons license, and indicate if changes were made. The Creative Commons Public Domain Dedication waiver (http://creativecommons.org/publicdomain/zero/1.0/) applies to the data made available in this article, unless otherwise stated. 
professionals to establish disease risks and develop interventions to address physical inactivity.

Questionnaires are typically used to assess physical activity level, as: 1) they are cost-effective and practical; 2) a large amount of information is collectable in a relatively short period of time; and 3) the results are easily quantifiable [9]. However, a number of disadvantages have been proposed, such as: 1) results are influenced by subjective measures; 2) misinterpretation of questions and recall bias due to language/cultural barriers or cognitive impairment; and 3) questionnaires not specifically developed for people with certain physiological/chronic conditions. Nonetheless, physical activity questionnaires are currently the most widely-used and acceptable forms of obtaining information on physical activity characteristics, particularly for larger-scale observational studies and research interventions [9]. There are several types of physical activity questionnaires which are primarily categorised according to recall periods. The two main recall methods currently utilised, measures recent physical activity performed over the past 7 days (i.e., Past-week PAQs) and the average week physical activity performed within the past 1-12 months (i.e., Usual-week PAQs) [10]. Previous research suggested that Past-week PAQs more accurately reflect the actual physical activity characteristics undertaken that week; however, Usual-week PAQs may minimise week-to-week variability [10], seasonal differences [11] and lifestyle factors, such as pregnancy [12]. Accordingly, the two recall methods may have distinct psychometric characteristics. It is therefore important to establish the validity and reliability of both types of PAQs, to ensure the PAQs selected are fit for purpose.

The Consensus-based Standards for the Selection of Health Measurement Instrument (COSMIN) checklist is a critical appraisal tool which evaluates methodological quality of studies that examine the psychometric properties of health related measures [13]. When combined with quality criteria for psychometric properties $[14,15]$, it provides a contemporary framework to assess overall psychometric quality of PAQs. According to a recent systematic review using the COSMIN checklist [16], when convergent validity was assessed by comparing PAQs with other measures (e.g., accelerometer or other PAQs), Past-week PAQs had higher correlations than Usual-week PAQs. These findings demonstrated that Past-week PAQs may assist clinicians in accessing the same constructs as those of other measures with better precision of PA level recordings. However, Doma and colleagues [16] only reported on studies that examined the psychometric properties of PAQs that were self-administered and excluded studies that administered PAQs via interviews. Whilst the ability to compare these data from self-administered PAQs to interview-administered PAQs are limited, it is currently the best available evidence of a similar construct.

The majority of PAQs can be either self-administered or interview-administered via face-to-face or telephone conducted by trained interviewers. For example, Active Australia Survey (AAS) is a commonly administered PAQ which assesses past-week PA level; its psychometric properties have previously been evaluated via both self-administration [17] and interview-administration [18]. The advantages of self-administered PAQs are that it is cost-effective, particularly when the PAQs distributed via postal mail or online, and minimises interviewer bias [19]. However, self-administered PAQs also risk introducing respondent bias, especially if respondents have literacy and numeracy difficulties [20]. These limitations can be overcome with interview-administered PAQs, although interviewees may overestimate reporting of their PA level due to social desirability [21]. In addition, the interviewee may over- or under-report physical activity level if instructions given by interviewers are not well standardised, or if interviewers are selective with phrasing the PAQs [22].

Although there is evidence that the mode of questionnaire administration may influence the accuracy and quality of the responses [20], to date, systematic reviews have only reported on the psychometric properties of self-administered PAQs [16, 23, 24], with overall findings indicating that only a few self-reported PAQs had reasonable reliability and validity ratings. No systematic reviews have explored the literature to determine the psychometric properties of interview-administered PAQs, particularly when compared between Past-week and Usual-week PAQs.

Therefore, the purpose of this systematic review was to evaluate the methodological quality of studies that have investigated the psychometric properties of interview-administered Past-week and Usual-week PAQs and to determine the overall psychometric quality for each PAQ. The results of this review will aid practitioners and researchers in selecting interview-administered PAQs that are appropriate for their purposes and through identifying the effects of recall differences on psychometric soundness.

\section{Methods}

The current systematic review was conducted in accordance with the Preferred Reporting Items for Systematic Reviews and Meta-Analyses (PRISMA) statement [25]. The PRISMA statement is a checklist that consists of 27 items that are used to ensure transparency of reporting for systematic reviews.

\section{Inclusion/exclusion criteria}

Studies on the psychometric properties of PAQs were only considered eligible if: 1) published in English; 2) physical 
activity questionnaires were developed in English; 3) administered to adults (>18yo) in English-speaking countries either with, or without pathological conditions (e.g., cardiovascular disease, musculoskeletal disease, metabolic disease or respiratory disease); 4) questionnaires consisted of recall methods of the past-week (i.e., previous 7 days) and usual-week (i.e., previous 7 days over 1-12 months); 5) questionnaires classified physical activity level based on energy expenditure, step count, distance travelled or duration of physical activity with the corresponding metabolic equivalent of task (MET); and 6) if the questionnaires were administered by trained interviewers. Studies were excluded if: 1) published as abstracts, 2) conference proceedings or dissertations; 3 ) used questionnaires with recall methods of less than 7 days, or recall over the previous 1-12 months that do not report average physical activity level over a 7-day period (i.e., average physical activity over the past month would be excluded whilst average 7-day physical activity over the past month would be included); 4) conducted using paediatric population or those with known cognitive impairment; 5) used questionnaires were translated into a language other than English; and 6) if the questionnaires were administered to individuals from non-English speaking backgrounds as cross-cultural validation was beyond the scope of this systematic review.

\section{Information sources}

A systematic literature search was conducted by two authors in June 2017 using two electronic databases (Embase and Pubmed). Subject headings and free text were used as part of the search for both databases, with date restrictions of the past half year applied for the free text search (refer to Table 1 for all search terms used during each electronic search). Following elimination of duplicates, a total of 7191 abstracts were retrieved from the search. The search process summary in accordance with the PRISMA guidelines is depicted in Fig. 1.

\section{Selection process}

Two independent reviewers with a health science background initially screened all abstracts against the pre-established inclusion/exclusion criteria, with each abstract rated as either meeting ("yes"), potentially meeting ("maybe") or not meeting ("no") the inclusion criteria. The reviewers were also trained by the primary author (KD) to ensure transparency of the inclusion criteria prior to abstract screening. Upon completion of abstract selection, a random $40 \%$ of abstracts were compared between the two independent reviewers and any disagreement between reviewers were consulted by a third reviewer (KD). Our calculation showed a Weighted Kappa calculation of 0.85 (95\%CI: 0.81-0.90) which was considered as excellent for inter-rater reliability [26]. Original articles from selected abstracts classified as either "yes" or "maybe" were accessed for further screening by the two reviewers using the same inclusion/ exclusion criteria. The first author provided final decisions if any disparity occurred between the two reviewers during the selection process of original articles.

\section{Assess study methodological quality using COSMIN ratings}

The COSMIN taxonomy of measurement properties and definitions for health-related patient-reported outcomes were used to evaluate the methodological quality of the included studies [27] (see Table 2). The COSMIN checklist evaluates the methodological quality of studies on psychometric properties and consists of nine domains: internal consistency, reliability (test-retest reliability,

Table 1 Search terms and databases used to obtain abstracts

\begin{tabular}{|c|c|c|}
\hline $\begin{array}{l}\text { Initial search: } \\
\text { Assessment retrieval }\end{array}$ & Database and Search Terms & Limitations \\
\hline \multirow[t]{2}{*}{ Subject Headings } & $\begin{array}{l}\text { Embase: (Questionnaire/) AND (Physical capacity/ OR "physical constitution and health"/ } \\
\text { OR "movement (physiology)"/ OR "physical activity, capacity and performance"/ OR } \\
\text { Exercise/ OR Performance/ OR Motor performance/) AND (Validation study/ OR validity/ OR } \\
\text { Psychometry/ OR Reliability/ OR Measurement accuracy/ OR measurement error/ OR } \\
\text { measurement precision/ OR measurement repeatability/) }\end{array}$ & $\begin{array}{l}\text { Humans; English; Adult: } \\
18 \text { to } 64 \text { years OR Aged: } 65+\text { years }\end{array}$ \\
\hline & $\begin{array}{l}\text { PubMed: ("Physical Conditioning, Human"[Mesh] OR "Physical Fitness"[Mesh] OR "Physical } \\
\text { Therapy Modalities"[Mesh] OR "Physical Endurance"[Mesh] OR "Physical Exertion"[Mesh] } \\
\text { OR "Exercise"[Mesh] OR "Motor Activity"[Mesh] OR "Exercise"[Mesh] OR "Exercise Movement } \\
\text { Techniques"[Mesh] OR "Exercise Therapy"[Mesh] OR "Psychomotor Performance"[Mesh] } \\
\text { OR "Motor Skills"[Mesh] OR "Motor Activity"[Mesh]) AND ("Surveys and Questionnaires } \\
\text { "[Mesh]) AND ("Psychometrics"[Mesh] OR "Reproducibility of Results"[Mesh] OR "Validation } \\
\text { Studies as Topic"[Mesh] OR "Bias (Epidemiology)"[Mesh] OR "Observer Variation"[Mesh]) }\end{array}$ & Humans; English; Adult: 19+ years \\
\hline \multirow[t]{2}{*}{ Free Text Words } & 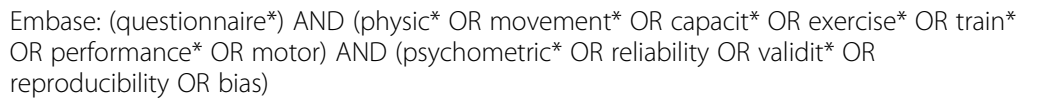 & $\begin{array}{l}\text { Publication date from } \\
2017 \text { - current }\end{array}$ \\
\hline & PubMed: As per Embase Free Text & $\begin{array}{l}\text { Publication date from } \\
2016 / 12 / 09 \text { to } 2017 / 06 / 09\end{array}$ \\
\hline
\end{tabular}




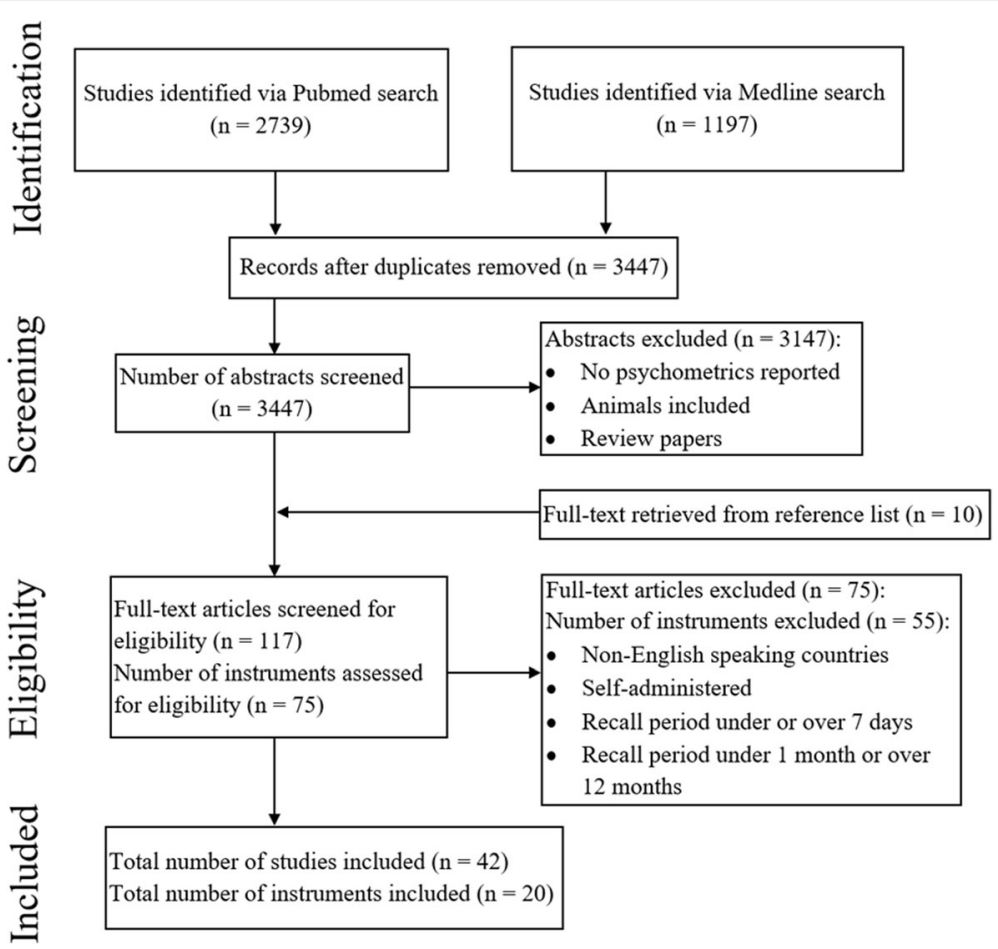

Fig. 1 Literature search conducted based on the PRISMA guidelines

Table 2 Definitions for aspects of domains and measurement properties from the COSMIN checklist by Mokkink et al. [27]

\begin{tabular}{|c|c|}
\hline $\begin{array}{l}\text { Psychometric } \\
\text { property }\end{array}$ & Domain: Definition \\
\hline & Validity: The degree to which an HR-PRO instrument measures the construct(s) it purports to measure \\
\hline Content validity & The degree to which the content of an HR-PRO instrument is an adequate reflection of the construct to be measured \\
\hline Face validity ${ }^{a}$ & $\begin{array}{l}\text { The degree to which an HR-PRO instrument indeed looks as though they are an adequate reflection of the construct } \\
\text { to be measured }\end{array}$ \\
\hline Construct validity & $\begin{array}{l}\text { The degree to which the scores of an HR-PRO instrument are consistent with hypotheses based on the assumption that a HR- } \\
\text { PRO instrument validly measures the construct to be measured }\end{array}$ \\
\hline $\begin{array}{l}\text { Structural } \\
\text { validity }^{b}\end{array}$ & $\begin{array}{l}\text { The degree to which the scores of an HR-PRO instrument are an adequate reflection of the dimensionality of the construct } \\
\text { to be measured }\end{array}$ \\
\hline $\begin{array}{l}\text { Hypothesis } \\
\text { testing }^{\text {b }}\end{array}$ & Item construct validity \\
\hline Criterion validity & The degree to which the scores of an instrument satisfactorily reflect a "gold standard" \\
\hline Responsiveness & Responsiveness: the capability of an HR-PRO instrument to detect change in the construct to be measured over time \\
\hline \multirow[t]{2}{*}{ Interpretability ${ }^{c}$} & Interpretability: the extent to which qualitative meaning is reflective of an instrument's quantitative scores or score change \\
\hline & Reliability: The degree to which the measurement is free from measurement error \\
\hline $\begin{array}{l}\text { Internal } \\
\text { consistency }\end{array}$ & The degree of the interrelatedness among the items \\
\hline Reliability & The proportion of total variance in the measurements due to "true" differences amongst patients \\
\hline Measuremen & The systematic and random error of a patient's score that is not attributed to true changes in the construct to be measured \\
\hline
\end{tabular}


inter-rater reliability and intra-rater reliability), measurement error (absolute measures), content validity, structural validity, hypothesis testing, cross-cultural validity, criterion validity and responsiveness [13]. 'Interpretability' is not considered to be a psychometric property; thus, it was excluded from this review. Of the nine domains, 'responsiveness' was not evaluated as the questionnaire's ability to detect changes over time was beyond the scope of this review. Furthermore, 'cross-cultural validity' was not assessed, as questionnaires either administered in non-English speaking countries or translated into non-English languages did not meet this review's inclusion criteria. Finally, whilst accelerometry and double-labelled water technique are considered the 'gold standard' of assessing physical activity level, there is a risk of under-reporting certain exercise modes (e.g., swimming or resistance training) [28]. Therefore, comparison of physical activity level reported by PAQs and objective measures (i.e., accelerometer and double-labelled water method) was considered as 'convergent validity'.

Each COSMIN checklist domain consists of between 5 to 18 items which addresses various aspects of study design and statistical methods. Based on a 4-point rating system (i.e., excellent, good, fair and poor, respectively), Terwee and colleagues [13] initially suggested that the overall methodological quality of each domain should mirror the rating of the lowest-rated item (i.e., if four items were rated 'Good' and one 'Poor', the overall score would be 'Poor'). However, given that each domain consists of items that assess a variety of methodological qualities, rating the overall methodological quality of a domain solely based on the lowest scoring single item undermines the ability of the checklist to explore subtle differences in psychometric qualities of each questionnaire [29]. Subsequently, a revised scoring method was implemented for this review by reporting the overall methodological quality of each domain as a percentage rating, as per Cordier, Speyer [29]. This revised scoring method has also been utilised successfully in a systematic review that compared Past-week and Usual-week PAQs, with sufficient sensitivity to detect differences between psychometric properties [16]. Specifically, the raw scores of each item were used to calculate a percentage of rating according to the following formula:

\section{Total score of each domain}

$$
=\frac{(\text { Total score obtained }- \text { minimum score obtained })}{(\text { Highest score possible }- \text { minimum score possible })} \times 100
$$

The final percentage score depicting the overall methodological quality of each domain was then classified as follows: Poor $=0-25.0 \%$, Fair $=25.1-50.0 \%$, Good $=50.1-75.0 \%$, Excellent $=75.1-100.0 \%$ [30]. Once the psychometric quality ratings of each paper were completed, ratings from a random $40 \%$ of papers were compared between two independent reviewers (KD and $L P$ ), resulting in a weighted Kappa of $0.84(0.62-1.00)$, indicating excellent agreement.

\section{Quality of the psychometric properties}

To account for varying sample sizes of each study when comparing the reliability (i.e., reproducibility) and convergent validity (a form of hypothesis testing that evaluates the correlation between two related measures, for example, physical activity levels measured from the PAQs under investigation and other measures) between PAQs, the weighted mean of correlation coefficient (i.e., $r$-values) were calculated, using the following formula:

$$
\bar{x}=\frac{\sum_{i=1}^{n} w_{i} x_{i}}{\sum_{i=1}^{n} w_{i}}
$$

Where $w=r$-value of the comparison within a study (e.g., PAQ vs. another instrument or PAQ vs. Accelerometer/pedometer) and $x=$ sample size of the comparison.

For the strength of reliability, once the weighted $r$-values were calculated for each study per PAQ, these measures were then averaged to compare the overall correlation between Past-week and Usual-week PAQs. For the strength of convergent validity, weighted $r$-values were averaged to compare overall correlations between Past-week and Usual-week PAQs, and between parameters that reported direct measures of PA level (e.g., diaries, other PAQs, accelerometers, pedometers) and indirect measures of PA level (e.g., aerobic fitness, muscular strength). If the sample size between each study was equivalent, then the normal non-weighted $r$-values were averaged. The strength of correlation was classified according to Cohen's method, with the following: $0-0.29,0.3-0.49$ and $\geq 0.5$ as weak, moderate and strong, respectively [31].

The psychometric quality of each measurement property per PAQ for each study (Table 3 ) was also classified using the following quality criteria: "positive" $(+)$, "conflicting" ( \pm ), "indeterminate" (?), "negative" (-), "not reported" (NR) or "not evaluated" (NE) [15, 30]. Studies that were rated as "poor" based on the COSMIN rating were excluded from further analyses and received "not evaluated" (NE). Finally, an overall quality score of assessments for each psychometric property was calculated based on the levels of evidence by Schellingerhout, Verhagen [14]. These scores were determined by integrating the methodological quality rating of the included studies on psychometric properties using the 
Table 3 The modified version of the psychometric quality rating set out by (Terwee et al., 2007) and (Cordier et al., [30])

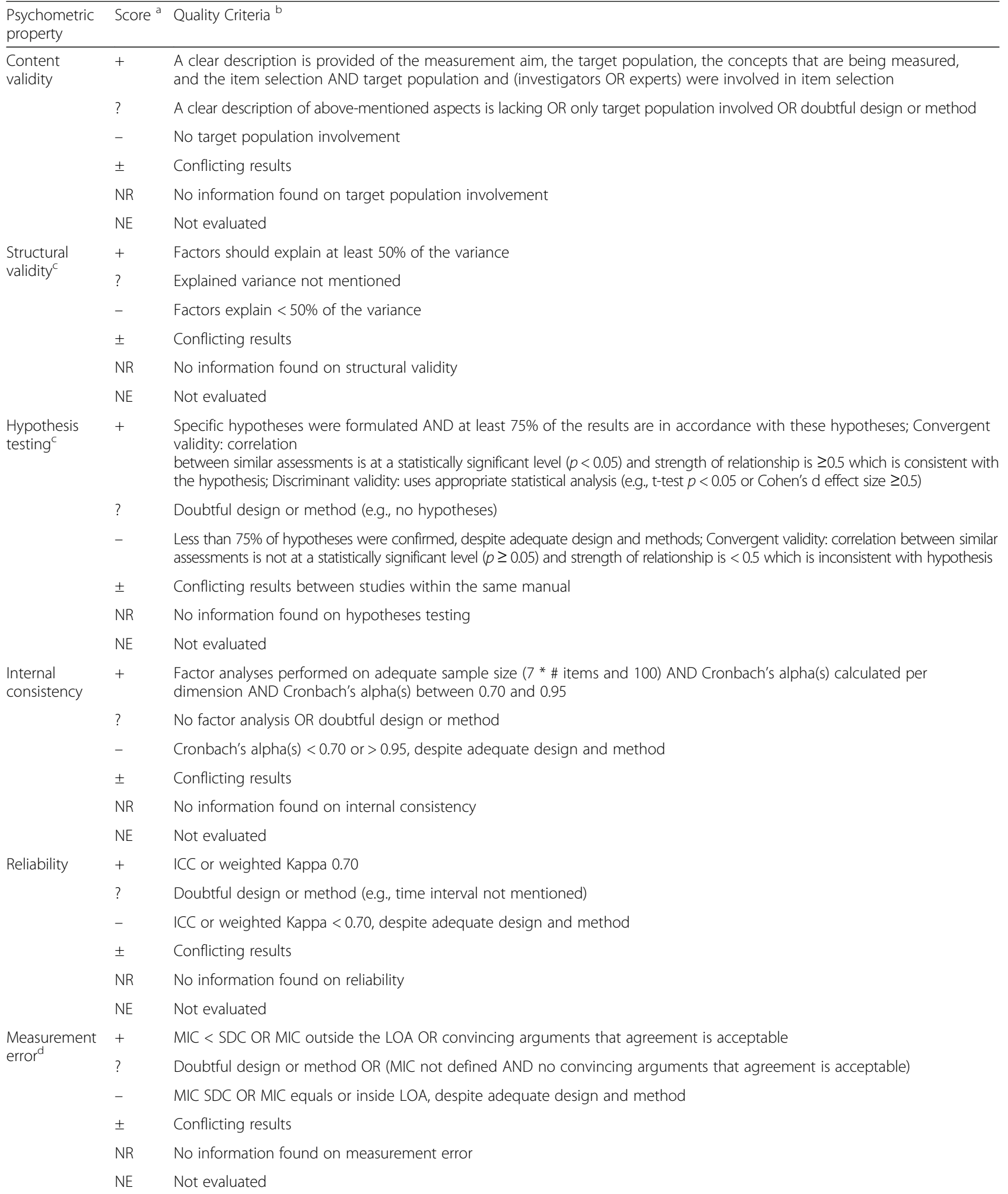

Notes. ${ }^{a}$ Scores: $+=$ positive rating, $?=$ indeterminate rating, $-=$ negative rating, $\pm=$ conflicting data, NR $=$ not reported, NE $=$ not evaluated (for study of poor methodological quality according to COSMIN rating, data are excluded from further evaluation

${ }^{b}$ Doubtful design or method is assigned when a clear description of the design or methods of the study is lacking, sample size smaller than 50 subjects (should be at least 50 in every subgroup analysis), or any important methodological weakness in the design or execution of the study

${ }^{c}$ Hypothesis testing: all correlations should be statistically significant (if not, these hypotheses are not confirmed) AND these correlations should be at least moderate ( $r>0.5$ )

${ }^{\mathrm{d}}$ Measurement error: MIC minimal important change, SDC smallest detectable change, LOA limits of agreement 
COSMIN checklist, and the quality criteria for measurement properties of assessment according to Terwee, Bot [15] and Cordier, Chen [30] (see Table 3). Figure 2 depicts a flowchart of the analysis process involved in determining the overall quality score for each assessment.

\section{Data items and synthesis of results}

Domains from the COSMIN checklist and psychometric property qualities were assessed for each included study according to Terwee, Bot [15] and Cordier, Chen [30]. The results were then reported in the following order: 1 ) the description of the literature search (see Table 1); 2) the characteristics of the interview-based PAQ measures (see Table 4) and studies reporting on the development and validation of the interview-based PAQ measures (see Table 4); 3) the methodological quality according to the COSMIN checklist of each study that have reported on the psychometric properties of PAQs (see Table 6); 4) the comparisons of the average weighted $r$-values of test-retest reliability and convergent validity between Past-week and Usual-week PAQs (see Table 7); 5) the quality of relevant psychometric properties for each study based on the criteria by Terwee, Bot [15] and Cordier, Chen [30] (Table 8); and 6) the overall quality rating of psychometric properties based on the levels of evidence by Schellingerhout, Verhagen [14] for each PAQ and comparing these results between Past-week and Usual-week PAQs (see Table 9).

\section{Results}

\section{Systematic literature search}

Following removal of duplicate abstracts, a total of 3447 abstracts were screened according to the inclusion criteria. Upon completion of screening, 75 PAQs and 117 of their corresponding full-text articles were examined for eligibility. Amongst these items, 20 PAQs and 42 of their corresponding articles were included. The remaining 55 PAQs were excluded for the following reasons: non-specified recall periods; recall period was beyond 7 days; recall period was less than 7 days; and various combinations of recall periods.

\section{Included physical activity questionnaires}

Table 4 displays the characteristics of the included PAQs, with description of their corresponding studies shown in Table 5. There were 6 PAQs that assessed Usual 7-days of PA level with two PAQs that had a 1-month recall period (Phone FITT and YPAS), one PAQ that had a 3-month recall period (IPEQ-WA) and three PAQs that had a 12-month recall period (CaMos, MAQ and NHS II; Table 4). The remaining 13 PAQs encompassed items that assessed PA level over the Past-7 days. A majority of PAQs had subscales that were separated by the intensity of PA (e.g., light, moderate and vigorous), whereas other PAQs had subscales categorised by the mode of PA (e.g., walking, stairs, occupational and gardening activities).

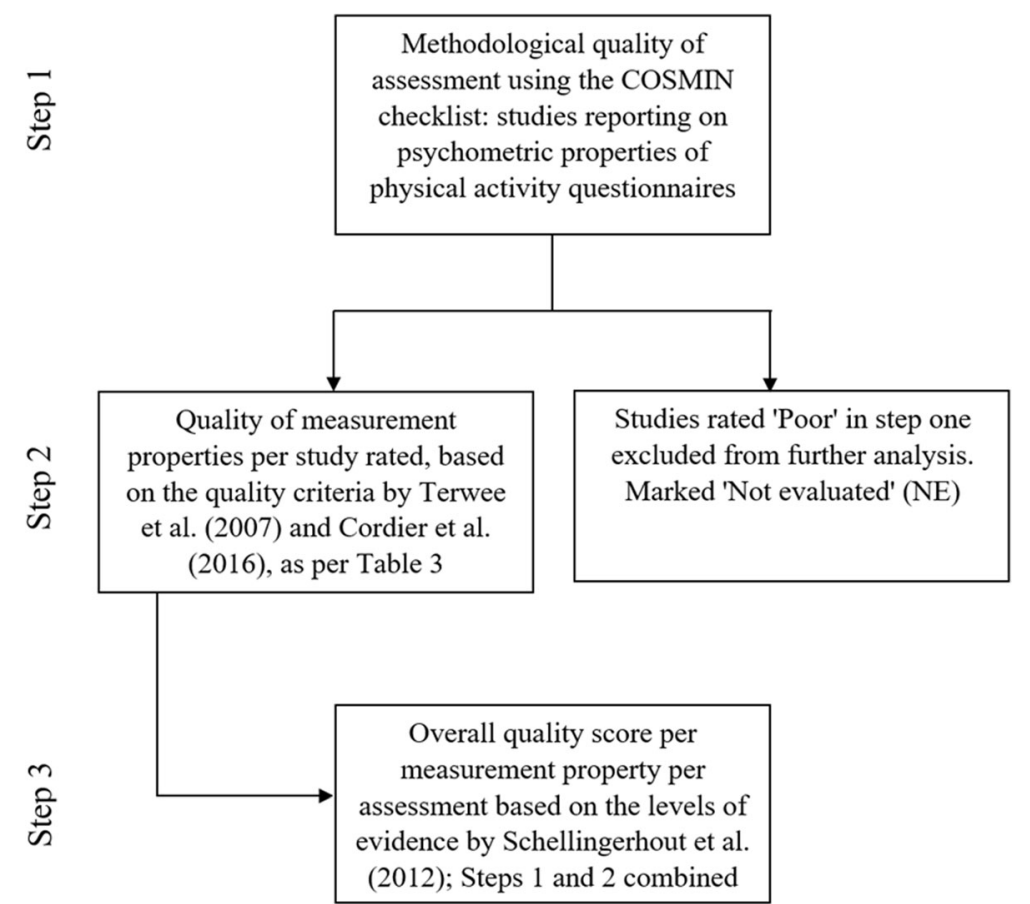

Fig. 2 Flow chart of the methodological quality rating based on COSMIN, derivation of the quality of psychometric properties and overall quality score per measurement 
Table 4 Characteristics of interview-administered Past-week and Usual-week physical activity questionnaires

\begin{tabular}{|c|c|c|c|c|c|c|}
\hline Instrument & Purpose of instrument & $\begin{array}{l}\text { Published } \\
\text { year }\end{array}$ & $\begin{array}{l}\text { Type of } \\
\text { administration/ } \\
\text { Recall method }\end{array}$ & $\begin{array}{l}\text { Number of } \\
\text { subscales/ } \\
\text { forms }\end{array}$ & $\begin{array}{l}\text { Total number } \\
\text { of items }\end{array}$ & Response options \\
\hline \multicolumn{7}{|c|}{ Usual-week Physical Activity Questionnaires } \\
\hline CaMos Usual-week & $\begin{array}{l}\text { To assess physical activity } \\
\text { among } \\
\text { those with susceptibility to } \\
\text { osteoporosis }\end{array}$ & 2004 & $\begin{array}{l}\text { Usual } 7 \text {-days } \\
\text { over } 12 \text { months }\end{array}$ & 4 & 10 & $\begin{array}{l}\text { Type of occupation: } \\
\text { - Full-time/Part-time/Unemployed/ } \\
\text { Disabled/Retired } \\
\text { - Mostly sitting/Mostly standing or } \\
\text { walking/Usually lift light loads/ } \\
\text { Usually lift heavy loads } \\
\text { Strenuousness of activity: } \\
\text { - Hours/week } \\
\text { Sitting activities: } \\
\text { - Hours/week } \\
\text { Sleep: } \\
\text { • Hours/day }\end{array}$ \\
\hline $\begin{array}{l}\text { IPEQ-WA Usual- } \\
\text { week }\end{array}$ & $\begin{array}{l}\text { To assess incidental physical } \\
\text { activity among older adults }\end{array}$ & 2010 & $\begin{array}{l}\text { Usual 7-days } \\
\text { over } 3 \text { months }\end{array}$ & 10 & 16 & $\begin{array}{l}\text { Type of activity: } \\
\text { - Minutes/week } \\
\text { Walking for exercise: } \\
\text { - Times/week } \\
\text { - Minutes/bout } \\
\text { Incidental walking: } \\
\text { - Times/week } \\
\text { - Minutes/bout } \\
\text { House maintenance/gardening: } \\
\text { - Minutes/day } \\
\text { Time on feet indoors: } \\
\text { - Minutes/day }\end{array}$ \\
\hline MAQ Usual-week & $\begin{array}{l}\text { To assess physical activity } \\
\text { among } \\
\text { the general population }\end{array}$ & 1990 & $\begin{array}{l}\text { Usual } 7 \text {-days } \\
\text { over } 12 \text { months }\end{array}$ & 6 & 9 & $\begin{array}{l}\text { Type of activity: } \\
\text { - Times/month } \\
\text { - Minutes/bout } \\
\text { Television viewing: } \\
\text { - Hours/day } \\
\text { Confined to bed or chair from injury/illness: } \\
\text { - Weeks/year } \\
\text { Difficulties with activities: } \\
\text { - Yes/no } \\
\text { Types of sports: } \\
\text { - Total years } \\
\text { Walk/cycle to work: } \\
\text { - Min/day }\end{array}$ \\
\hline $\begin{array}{l}\text { NHS II Usual 7- } \\
\text { days }\end{array}$ & $\begin{array}{l}\text { To assess the health conditions } \\
\text { of } \\
\text { nurses working at hospitals }\end{array}$ & 1989 & $\begin{array}{l}\text { Usual } 7 \text {-days } \\
\text { over } 12 \text { months }\end{array}$ & 3 & 16 & $\begin{array}{l}\text { Daily flights of stairs: } \\
\cdot \leq 2 ; 3-4 ; 5-9 ; 10-14 ; \geq 15 \\
\text { Physical activity per week: } \\
\cdot 0-11+\text { hours } \\
\text { Sedentary time per week: } \\
\cdot 0-90+\text { hours }\end{array}$ \\
\hline $\begin{array}{l}\text { Phone-FITT Usual- } \\
\text { week }\end{array}$ & $\begin{array}{l}\text { To assess physical activity } \\
\text { among } \\
\text { older adults via phone }\end{array}$ & 2008 & $\begin{array}{l}\text { Usual 7-days } \\
\text { over } 1 \text { month }\end{array}$ & 9 & 16 & $\begin{array}{l}\text { Type of household activity: } \\
\text { - Hours/week } \\
\text { Type of recreational activity: } \\
\text { - Hours/week } \\
\text { Type of seasonal recreational activity: } \\
\text { - Hours/week } \\
\text { Other physical activity: } \\
\text { - Hours/week }\end{array}$ \\
\hline YPAS Usual 7-days & $\begin{array}{l}\text { To assess physical activity } \\
\text { among } \mathrm{V} \\
\text { older adults }\end{array}$ & 1993 & $\begin{array}{l}\text { Usual 7-days } \\
\text { over } 1 \text { month }\end{array}$ & 6 & 39 & $\begin{array}{l}\text { Type of activity: } \\
\text { - Hours/week } \\
\text { Vigorousness of activity: } \\
\text { - Frequency /week or /month } \\
\text { Leisurely walk: } \\
\text { - Frequency /week or /month; Duration in } \\
\text { minutes } \\
\text { General movement: } \\
\text { - Hours/day } \\
\text { Standing and sitting: } \\
\text { - Hours/day } \\
\text { Seasonal changes: } \\
\text { - Compare current season }\end{array}$ \\
\hline \multicolumn{7}{|c|}{ Past-week Physical Activity Questionnaires } \\
\hline AAS Past 7-days & $\begin{array}{l}\text { Population surveillance of } \\
\text { physical } \\
\text { activity in Australian adults }\end{array}$ & 2003 & Past 7-days & 4 & 8 & $\begin{array}{l}\text { Walking activities: } \\
\text { - Frequency/week; Hours/week; } \\
\text { minutes/week } \\
\text { Vigorous yard work: }\end{array}$ \\
\hline
\end{tabular}


Table 4 Characteristics of interview-administered Past-week and Usual-week physical activity questionnaires (Continued)

\begin{tabular}{|c|c|c|c|c|c|c|}
\hline Instrument & Purpose of instrument & $\begin{array}{l}\text { Published } \\
\text { year }\end{array}$ & $\begin{array}{l}\text { Type of } \\
\text { administration/ } \\
\text { Recall method }\end{array}$ & $\begin{array}{l}\text { Number of } \\
\text { subscales/ } \\
\text { forms }\end{array}$ & $\begin{array}{l}\text { Total number } \\
\text { of items }\end{array}$ & Response options \\
\hline & & & & & & $\begin{array}{l}\text { - Frequency/week; Hours/week; } \\
\text { minutes/week } \\
\text { Vigorous activities other than yard } \\
\text { work: } \\
\text { - Frequency/week; Hours/week; } \\
\text { minutes/week } \\
\text { Moderate activities: } \\
\text { - Frequency/week; Hours/week; } \\
\text { minutes/week }\end{array}$ \\
\hline AAS (modified) & $\begin{array}{l}\text { Population surveillance of } \\
\text { physical } \\
\text { activity in Australian adults }\end{array}$ & 2013 & Past 7-days & 4 & 8 & $\begin{array}{l}\text { Walking activities: } \\
\text { - Frequency/week; Hours/week; } \\
\text { minutes/week } \\
\text { Vigorous yard work: } \\
\text { - Frequency/week; Hours/week; } \\
\text { minutes/week } \\
\text { Vigorous activities other than yard work: } \\
\text { - Frequency/week; Hours/week; } \\
\text { minutes/week } \\
\text { Moderate activities: } \\
\text { - Frequency/week; Hours/week; } \\
\text { minutes/week }\end{array}$ \\
\hline $\begin{array}{l}\text { CAQ-PAI Past 7- } \\
\text { days }\end{array}$ & $\begin{array}{l}\text { To measure overall kilocalories } \\
\text { expended in leisure-time phys- } \\
\text { ical } \\
\text { activity }\end{array}$ & 1978 & Past 7-days & 3 & 4 & $\begin{array}{l}\text { Walking: } \\
\text { - Blocks/day } \\
\text { Stairs: } \\
\text { - Flights/day } \\
\text { Recreational activities: } \\
\text { - Frequency/week; Hours/week; } \\
\text { minutes/session }\end{array}$ \\
\hline $\begin{array}{l}\text { Checklist } \\
\text { Questionnaire Past } \\
\text { 7-days }\end{array}$ & $\begin{array}{l}\text { Assess the frequency and } \\
\text { duration of physical activities } \\
\text { performed in the previous } 7 \\
\text { days }\end{array}$ & 2012 & Past 7-days & 10 & 64 & $\begin{array}{l}\text { Household activities: } \\
\text { - Frequency/week; Hours/week; } \\
\text { minutes/week } \\
\text { Yard activities: } \\
\text { - Frequency/week; Hours/week; } \\
\text { minutes/week } \\
\text { Family activities: } \\
\text { - Frequency/week; Hours/week; } \\
\text { minutes/week } \\
\text { Community/volunteer/church: } \\
\text { - Frequency/week; Hours/week; } \\
\text { minutes/week } \\
\text { Transportation: } \\
\text { - Frequency/week; Hours/week; } \\
\text { minutes/week } \\
\text { Miscellaneous: } \\
\text { - Frequency/week; Hours/week; } \\
\text { minutes/week } \\
\text { Other time: } \\
\text { Exercise, sports and dancing: } \\
\text { - Frequency/week; Hours/week; } \\
\text { minutes/week } \\
\text { Employment: } \\
\text { - Frequency/week; Hours/week; } \\
\text { minutes/week } \\
\text { Miscellaneous: } \\
\text { - Frequency/week; Hours/week; } \\
\text { minutes/week }\end{array}$ \\
\hline $\begin{array}{l}\text { Global } \\
\text { Questionnaire }\end{array}$ & $\begin{array}{l}\text { To assess physical activity } \\
\text { among } \\
\text { older adults }\end{array}$ & 2001 & Past 7-days & 5 & 35 & $\begin{array}{l}\text { Type of activity: } \\
\text { - Hours/week } \\
\text { Vigorousness of activity: } \\
\text { - Frequency/week or /month } \\
\text { Leisurely walk: } \\
\text { - Frequency/week or /month; } \\
\text { Duration in minutes } \\
\text { General movement: } \\
\text { - Hours/day }\end{array}$ \\
\hline $\begin{array}{l}\text { IPAQ-LF Past } \\
\text { 7-days }\end{array}$ & As for IPAQ-LF (Telephone) & 2002 & Past 7-days & $\begin{array}{l}\text { As for IPAQ-LF } \\
\text { (Telephone) }\end{array}$ & $\begin{array}{l}\text { As for IPAQ-LF } \\
\text { (Telephone) }\end{array}$ & $\begin{array}{l}\text { Vigorous activities } \\
\text { - Days/week; Hours/week; minutes/ } \\
\text { day } \\
\text { Moderate activities } \\
\text { - Days/week; Hours/week; minutes/ } \\
\text { day } \\
\text { Light activities }\end{array}$ \\
\hline
\end{tabular}


Table 4 Characteristics of interview-administered Past-week and Usual-week physical activity questionnaires (Continued)

\begin{tabular}{|c|c|c|c|c|c|c|}
\hline Instrument & Purpose of instrument & $\begin{array}{l}\text { Published } \\
\text { year }\end{array}$ & $\begin{array}{l}\text { Type of } \\
\text { administration/ } \\
\text { Recall method }\end{array}$ & $\begin{array}{l}\text { Number of } \\
\text { subscales/ } \\
\text { forms }\end{array}$ & $\begin{array}{l}\text { Total number } \\
\text { of items }\end{array}$ & Response options \\
\hline & & & & & & $\begin{array}{l}\text { - Days/week; Hours/week; minutes/ } \\
\text { day } \\
\text { Sitting time } \\
\text { - Days/week; Hours/week; minutes/ } \\
\text { day }\end{array}$ \\
\hline $\begin{array}{l}\text { IPAQ-SF Past } \\
\text { 7-days }\end{array}$ & $\begin{array}{l}\text { As for IPAQ-LF (Telephone } \\
\text { version) }\end{array}$ & 2002 & Past 7-days & 4 & 7 & $\begin{array}{l}\text { Vigorous activities } \\
\text { - Days/week; Hours/week; minutes/ } \\
\text { day } \\
\text { Moderate activities } \\
\text { - Days/week; Hours/week; minutes/ } \\
\text { day } \\
\text { Light activities } \\
\text { - Days/week; Hours/week; minutes/ } \\
\text { day } \\
\text { Sitting time } \\
\text { - Days/week; Hours/week; minutes/ } \\
\text { day }\end{array}$ \\
\hline NZPAQ-LF & $\begin{array}{l}\text { Retrospective diary that } \\
\text { assesses all } \\
\text { dimensions of physical activity } \\
\text { type } \\
\text { and level in New Zealand }\end{array}$ & 2008 & Past 7-days & 5 & 11 & $\begin{array}{l}\text { Sport/recreation: } \\
\text { - Days/week; Hours/week; minutes/ } \\
\text { day } \\
\text { Transport: } \\
\text { - Days/week; Hours/week; minutes/ } \\
\text { day } \\
\text { Occupation: } \\
\text { - Days/week; Hours/week; minutes/ } \\
\text { day } \\
\text { Cultural/incidental activities: } \\
\text { - Days/week; Hours/week; minutes/ } \\
\text { day } \\
\text { Inactivity: } \\
\text { - Days/week; Hours/week; minutes/ } \\
\text { day }\end{array}$ \\
\hline NZPAQ-SF & $\begin{array}{l}\text { Modified version of IPAQ-SF to } \\
\text { as } \\
\text { sess physical activity level } \\
\text { whilst } \\
\text { reflecting the culture in } \\
\text { New Zealand }\end{array}$ & 2008 & Past 7-days & 4 & 7 & $\begin{array}{l}\text { Walking activities: } \\
\text { - Days/week; Hours/week; minutes/ } \\
\text { day } \\
\text { Moderate physical activity: } \\
\text { - Days/week; Hours/week; minutes/ } \\
\text { day } \\
\text { Vigorous physical activity } \\
\text { - Days/week; Hours/week; minutes/ } \\
\text { day: } \\
\text { Frequency of activity: } \\
\text { - Days/week; Hours/week; minutes/ } \\
\text { day }\end{array}$ \\
\hline PAAQ & $\begin{array}{l}\text { To assess physical activity level } \\
\text { in } \\
\text { line with the Canadian Physical } \\
\text { Activity Guidelines }\end{array}$ & 2015 & Past 7-days & 4 & 12 & $\begin{array}{l}\text { Walking or cycling to destination } \\
\text { - Yes/No } \\
\text { - Days/week; Hours/week; minutes/ } \\
\text { day } \\
\text { Recreational activities, organised } \\
\text { activities lasting minimum of } 10 \text { min that } \\
\text { caused sweat or hard breathing: } \\
\text { - Yes/No } \\
\text { - Days/week; Hours/week; minutes/ } \\
\text { day } \\
\text { Activities at work, home or } \\
\text { volunteering that caused sweat or hard } \\
\text { breathing: } \\
\text { - Yes/No } \\
\text { - Days/week; Hours/week; minutes/ } \\
\text { day } \\
\text { Vigorous exercises that caused } \\
\text { breathlessness: } \\
\text { - Yes/No } \\
\text { - Days/week; Hours/week; minutes/ } \\
\text { day }\end{array}$ \\
\hline PASE Past 7-days & $\begin{array}{l}\text { To assess leisure, occupational } \\
\text { and } \\
\text { household physical activities } \\
\text { amongst the elderly }\end{array}$ & 1991 & Past 7-days & 3 & 27 & $\begin{array}{l}\text { Recreational activities: } \\
\text { - Frequency/week; < } 1 \mathrm{~h}, 1-2 \mathrm{~h}, 2-4 \mathrm{~h} \\
\text { or }>4 \mathrm{~h} \\
\text { Household activities: } \\
\text { - Yes or no; Type of activities } \\
\text { Occupational activities: } \\
\text { - Hours/week; Type of activities }\end{array}$ \\
\hline
\end{tabular}


Table 4 Characteristics of interview-administered Past-week and Usual-week physical activity questionnaires (Continued)

\begin{tabular}{|c|c|c|c|c|c|c|}
\hline Instrument & Purpose of instrument & $\begin{array}{l}\text { Published } \\
\text { year }\end{array}$ & $\begin{array}{l}\text { Type of } \\
\text { administration/ } \\
\text { Recall method }\end{array}$ & $\begin{array}{l}\text { Number of } \\
\text { subscales/ } \\
\text { forms }\end{array}$ & $\begin{array}{l}\text { Total number } \\
\text { of items }\end{array}$ & Response options \\
\hline PWMAQ & $\begin{array}{l}\text { To assess leisure physical } \\
\text { activities } \\
\text { during the past week }\end{array}$ & 2009 & Past 7-days & 6 & 9 & $\begin{array}{l}\text { Type of activity: } \\
\text { - Times/week } \\
\text { - Minutes/bout } \\
\text { Television viewing: } \\
\text { - Hours/day } \\
\text { Confined to bed or chair from } \\
\text { injury/lllness: } \\
\text { - Minutes/week } \\
\text { Difficulties with activities: } \\
\text { - Yes/no } \\
\text { Types of sports: } \\
\text { - Total years } \\
\text { Walk/cycle to work: } \\
\text { - Min/day }\end{array}$ \\
\hline PAR Past 7-days & $\begin{array}{l}\text { To assess sleep and physical } \\
\text { activity } \\
\text { patterns }\end{array}$ & 1985 & Past 7-days & 6 & 15 & $\begin{array}{l}\text { Occupational activities: } \\
\text { - Yes or No; Frequency/week; Hours/ } \\
\text { week; Days/week } \\
\text { Moderate, Hard and Very Hard in the Morning: } \\
\text { - Minutes } \\
\text { Moderate, Hard and Very Hard in the Afternoon: } \\
\text { - Minutes } \\
\text { Moderate, Hard and Very Hard in } \\
\text { the Evening: } \\
\text { Strength: } \\
\text { - Minutes } \\
\text { Flexibility: } \\
\text { - Minutes }\end{array}$ \\
\hline $\begin{array}{c}\text { VAPAQ } \\
\text { Past 7-days }\end{array}$ & $\begin{array}{l}\text { To measures physical activities } \\
\text { amongst veterans }\end{array}$ & 2003 & Past 7-days & 3 & 6 & $\begin{array}{l}\text { Walking activities: } \\
\text { - Blocks/day } \\
\text { Sports/recreational activities: } \\
\text { - Frequency/week; Hours/week; } \\
\quad \text { minutes/session } \\
\text { Occupational activities: } \\
\text { - Frequency/week; Hours/week; minutes/session }\end{array}$ \\
\hline
\end{tabular}

CaMos Canadian Multicentre Osteoporosis Study, IPEQ-WA Incidental and Planned Exercise Questionnaire, MAQ Modified Activity Questionnaire, NHS /I Nurses' Health Study version II, Phone-FITT Phone Fitness, YPAS Yale Physical Activity Survey, AAS Active Australia Survey, CAQ-PAI College Alumni Questionnaire - Physical Activity Index, IPAQ-LF International Physical Activity Questionnaire Long Form, IPAQ-SF International Physical Activity Questionnaire Short Form, NZPAQ-LF New Zealand Physical Activity Questionnaire Long Form, NZPAQ-SF New Zealand Physical Activity Questionnaire Short Form, PAAQ Physical Activity Adult Questionnaire, PASE Physical Activity Scale for the Elderly, PWMAQ Past Week Modified Activity Questionnaire, PAR Physical Activity Recall Questionnaire, VAPAQ Veterans Physical Activity Questionnaire

\section{Psychometric properties of PAQs}

Table 6 provides an overview of the methodological quality assessment of studies reporting on psychometric properties of usual-week and past-week physical activity questionnaires using the COSMIN checklist. The most frequently reported psychometric properties based on the COSMIN rating assessment was hypothesis testing (18 of 20 PAQs), ranging from fair to excellent qualities, followed by reliability (13 of 20 PAQs), ranging from good to excellent qualities. The least reported psychometric properties included measurement error (4 of 20 PAQs), ranging from good to excellent qualities, internal consistency (3 of $20 \mathrm{PAQs),} \mathrm{ranging} \mathrm{from} \mathrm{poor} \mathrm{to} \mathrm{fair}$ qualities and content validity (3 of 20 PAQs), ranging from fair to good qualities. No studies were identified that reported structural validity. When different PAQ recall methods were compared (i.e., Past-week PAQ versus Usual-week PAQ), similar frequencies in psychometric properties were found for Usual 7-day PAQs and Past 7-day PAQs with internal consistency (16.7 and $14.3 \%$, respectively) and content validity (16.7 and 14.3\%, respectively). However, notable differences were also shown with Usual 7-day PAQs more frequently reported for reliability (83.3\% vs. 57.1\%) and Past 7-day PAQs more frequently for measurement error (24.1\% vs. 16.7\%) and hypothesis testing ( $92.9 \%$ vs. $83.3 \%$ ).

Table 7 demonstrates the weighted mean of the $r$-values for test-retest reliability and convergent validity between the types of PAQ (i.e., Usual-week vs. Past-week) and type of comparator measures (i.e., direct vs. indirect measures). Test-retest reliability data was available for 7 of the 20 PAQs. According to the average weighted mean of the $r$-values, the reliability of both Usual-week and Past-week PAQs showed strong correlations when assessed across two separate time points, with similar reliability measures for Usualweek $(r=0.63)$ and Past-week $(r=0.56) \quad$ PAQs. According to Cohen's methods, when direct and indirect measures were combined for convergent validity (data was available for 17 of 20 PAQs), Usual-week PAQs exhibited a moderate correlation $(r=0.30)$, whereas Past-week PAQs shows a weak correlation $(r=0.28)$. With 
Table 5 Description of studies for the development and validation of interview-administered Past-week and Usual-week physical activity questionnaires

\begin{tabular}{|c|c|c|c|c|c|}
\hline Instrument & Reference & Purpose of study & Study population & Health condition & $\begin{array}{l}\text { Age range (R; mean } \pm \\
\text { standard deviation) }\end{array}$ \\
\hline \multicolumn{6}{|c|}{ Usual-week Physical Activity Questionnaires } \\
\hline $\begin{array}{c}\text { CaMos } \\
\text { Usual-week }\end{array}$ & $\begin{array}{l}\text { Nadalin, } \\
\text { Bentvelsen } \\
\text { [45] }\end{array}$ & $\begin{array}{l}\text { To assess test-retest reliability of a } \\
\text { portion of the CaMos question- } \\
\text { naire using a combination of ad- } \\
\text { ministration modes }\end{array}$ & Reliability $(N=367)$ & $\begin{array}{l}\text { Physical: healthy with } \\
\text { possible osteoporosis } \\
\text { Cognitive: Not screened }\end{array}$ & Total sample: $R=45-80$ (NR)y \\
\hline $\begin{array}{l}\text { IPEQ-WA } \\
\text { Usual-week }\end{array}$ & $\begin{array}{l}\text { Merom, } \\
\text { Delbaere } \\
{[46]}\end{array}$ & $\begin{array}{l}\text { Assessed construct validity and } \\
\text { responsiveness of IPEQ }\end{array}$ & $\begin{array}{l}\text { Male (I) \& Female (II): } \\
\text { Validity }(N=40 \text { \& 86) }\end{array}$ & $\begin{array}{l}\text { Physical: No chronic } \\
\text { disease conditions } \\
\text { Cognitive: Healthy based } \\
\text { on cognitive test }\end{array}$ & $\begin{array}{l}\text { Total sample: } \mathrm{R}=\mathrm{NR} \text {; (I) NR; (II) } \\
\mathrm{NR}\end{array}$ \\
\hline \multirow[t]{4}{*}{$\begin{array}{c}\text { MAQ } \\
\text { Usual-week }\end{array}$} & $\begin{array}{l}\text { Pettee } \\
\text { Gabriel, } \\
\text { McClain } \\
{[47]}\end{array}$ & $\begin{array}{l}\text { Test-retest reliability and } \\
\text { convergent validity of five PAQs } \\
\text { commonly used in larger health } \\
\text { studies involving middle-aged } \\
\text { women }\end{array}$ & $\begin{array}{l}\text { Female (I): } \\
\text { Repeatability \& } \\
\text { Validity }(N=62-66)\end{array}$ & $\begin{array}{l}\text { Physical: No chronic } \\
\text { disease conditions } \\
\text { Cognitive: Not screened }\end{array}$ & $\begin{array}{l}\text { Total sample: } R=45-65(52.6 \\
\pm 5.4) y\end{array}$ \\
\hline & $\begin{array}{l}\text { Kriska, } \\
\text { Knowler } \\
{[48]}\end{array}$ & $\begin{array}{l}\text { To examine the reliability and } \\
\text { validity of the MAQ }\end{array}$ & $\begin{array}{l}\text { Male (I) \& Female }(I): \\
\text { Repeatability }(N=69) \\
\text { Validity }(N=21)\end{array}$ & $\begin{array}{l}\text { Physical: No physical } \\
\text { limitations with possible } \\
\text { type II diabetes mellitus } \\
\text { Cognitive: Not screened }\end{array}$ & $\begin{array}{l}\text { Total sample: } R=10-59 \\
\text { (NR)yr.; (I) 10-59 NR; (II) 10-59 } \\
\text { (NR)y }\end{array}$ \\
\hline & $\begin{array}{l}\text { Kriska, } \\
\text { Edelstein } \\
\text { [49] }\end{array}$ & $\begin{array}{l}\text { To compare MAQ with other PAQs } \\
\text { among individuals with type } 2 \\
\text { diabetes }\end{array}$ & $\begin{array}{l}\text { Male: Validity (I) (N= } \\
\text { 1043) } \\
\text { Female: Validity (II) (N } \\
=2191)\end{array}$ & $\begin{array}{l}\text { Physical: No physical } \\
\text { limitations with possible } \\
\text { type II diabetes mellitus } \\
\text { Cognitive: Not screened }\end{array}$ & $\begin{array}{l}\text { Total sample: NR }(50.6 \pm \\
\text { 10.7)yr.; (I) NR; (II) NR }\end{array}$ \\
\hline & $\begin{array}{l}\text { Schulz, } \\
\text { Harper [50] }\end{array}$ & $\begin{array}{l}\text { To compare MAQ with direct } \\
\text { measures of energy expenditure }\end{array}$ & $\begin{array}{l}\text { Male: Validity (I) (N= } \\
\text { 12) } \\
\text { Female: Validity (II) (N } \\
=9 \text { ) }\end{array}$ & $\begin{array}{l}\text { Physical: No physical } \\
\text { limitations with possible } \\
\text { type II diabetes mellitus } \\
\text { Cognitive: Not screened }\end{array}$ & $\begin{array}{l}\text { Total sample: } N R ; \text { (I) } R=N R \\
(35.4 \pm 13.8) y r ; \text { (II) } R=N R \\
(31.3 \pm 13.0) y\end{array}$ \\
\hline $\begin{array}{c}\text { NHS II } \\
\text { Usual-week }\end{array}$ & $\begin{array}{l}\text { Pettee } \\
\text { Gabriel, } \\
\text { McClain } \\
\text { [47] }\end{array}$ & As for MAQ & As for MAQ & As for MAQ & As for MAQ \\
\hline $\begin{array}{l}\text { Phone-FITT } \\
\text { Usual-week }\end{array}$ & $\begin{array}{l}\text { Gill, Jones } \\
\text { [51] }\end{array}$ & $\begin{array}{l}\text { To develop the Phone-FITT and to } \\
\text { evaluate the test-retest reliability } \\
\text { and criterion-related (concurrent) } \\
\text { and construct (convergent, dis- } \\
\text { criminant and known-groups) } \\
\text { validity }\end{array}$ & $\begin{array}{l}\text { Male: Repeatability (I) } \\
\text { \& Validity (II) (N=22 \& } \\
\text { 12) } \\
\text { Female: Repeatability } \\
\text { (III) \& Validity (IV) ( } N= \\
21 \text { \& 36) }\end{array}$ & $\begin{array}{l}\text { Physical: No chronic } \\
\text { disease conditions } \\
\text { Cognitive: Not screened }\end{array}$ & $\begin{array}{l}\text { Total sample: } \mathrm{R}=73-87(79.4 \\
\pm 2.9) \mathrm{y} ;(\text { I) } 76-86(79.4 \pm 3.2) \mathrm{y} ; \\
\text { (II) } 72-82(76.5 \pm 3.4) ;(\text { III) } 76- \\
86(79.5 \pm 2.7) \mathrm{y} ;(\text { (IV) } 71-89 \\
(77.8 \pm 5.1) \mathrm{y}\end{array}$ \\
\hline \multirow[t]{4}{*}{$\begin{array}{c}\text { YPAS } \\
\text { Usual-week }\end{array}$} & $\begin{array}{l}\text { Colbert, } \\
\text { Matthews } \\
\text { [52] }\end{array}$ & $\begin{array}{l}\text { Compared validity of a variety of } \\
\text { physical activity measurement } \\
\text { tools in older adults }\end{array}$ & Validity $(N=56)$ & $\begin{array}{l}\text { Physical: Musculoskeletal } \\
\text { conditions, lung disease, } \\
\text { cancer and hypertension } \\
\text { Cognitive: Not screened }\end{array}$ & $\begin{array}{l}\text { Total sample: } R=N R(74.7 \pm \\
6.5) y\end{array}$ \\
\hline & $\begin{array}{l}\text { Dipietro, } \\
\text { Caspersen } \\
{[53]}\end{array}$ & $\begin{array}{l}\text { Preliminary repeatability data and } \\
\text { validation results relative to } \\
\text { selected physiologic variables }\end{array}$ & $\begin{array}{l}\text { Male (I) \& Female (II): } \\
\text { Repeatability }(N=20 \\
\text { \& 56); Validity }(N=14 \\
\text { \& 11) }\end{array}$ & $\begin{array}{l}\text { Physical: No chronic } \\
\text { disease conditions } \\
\text { Cognitive: Not screened }\end{array}$ & $\begin{array}{l}\text { Total sample: } \mathrm{R}=\mathrm{NR}(71.0 \pm \\
\text { 6.6)y; (I) } \mathrm{R}=\mathrm{NR}(70.9 \pm 6.2) \mathrm{y} ; \\
\text { (II) } \mathrm{R}=\mathrm{NR}(69.6 \pm 6.0) \mathrm{y}\end{array}$ \\
\hline & $\begin{array}{l}\text { Gennuso, } \\
\text { Matthews } \\
{[54]}\end{array}$ & $\begin{array}{l}\text { Reliability and validity of physical } \\
\text { activity surveys for assessing time } \\
\text { spent in sedentary behavior in } \\
\text { older adults }\end{array}$ & $\begin{array}{l}\text { Validity \& } \\
\text { Repeatability }(N=58)\end{array}$ & $\begin{array}{l}\text { Physical: NR } \\
\text { Cognitive: Not screened }\end{array}$ & $\begin{array}{l}\text { Total sample: } R=66-88(75.1 \\
\pm 6.5) y\end{array}$ \\
\hline & $\begin{array}{l}\text { Harada, } \\
\text { Chiu [55] }\end{array}$ & $\begin{array}{l}\text { Assess the known-groups and con- } \\
\text { struct validity of CHAMPS, PASE } \\
\text { and YPAS }\end{array}$ & $\begin{array}{l}\text { Retirement homes (I) \& } \\
\text { Community centres (II): } \\
\text { Validity }(N=36 \& 51)\end{array}$ & $\begin{array}{l}\text { Physical: Musculoskeletal } \\
\text { conditions, lung disease, } \\
\text { diabetes and } \\
\text { hypertension } \\
\text { Cognitive: Healthy based } \\
\text { on cognitive test }\end{array}$ & $\begin{array}{l}\text { Total sample: } R=56-89(75.0 \\
\pm 6.0) ;(I) R=65-89(79.0 \pm \\
6.0) ;(\text { II) } R=65-86(73.0 \pm 5.0)\end{array}$ \\
\hline
\end{tabular}

Kolbe- $\quad$ Validity and reliability of the YPAS Alexander, and the short version of the Lambert IPAQ in older South African adults [56]
Male (I) \& Female (II): $(N=52$ \& 70); Sample (N) not reported between psychometric
Physical: NR

Total sample: $R=62-69(66 \pm$ Cognitive: Not screened NR)y; (I) 62-69 (67 \pm NR); (II) 62-69 (65 $\pm N R)$ 
Table 5 Description of studies for the development and validation of interview-administered Past-week and Usual-week physical activity questionnaires (Continued)

\begin{tabular}{|c|c|c|c|c|c|}
\hline Instrument & Reference & Purpose of study & Study population & Health condition & $\begin{array}{l}\text { Age range ( } \mathrm{R} \text {; mean } \pm \\
\text { standard deviation) }\end{array}$ \\
\hline \multicolumn{6}{|c|}{ measures } \\
\hline & $\begin{array}{l}\text { Moore, Ellis } \\
{[57]}\end{array}$ & $\begin{array}{l}\text { Construct validity of four PAQs in } \\
\text { culturally diverse older adults }\end{array}$ & $\begin{array}{l}\text { African American (I) \& } \\
\text { Caucasian (II): Validity } \\
(N=54)\end{array}$ & $\begin{array}{l}\text { Physical: Musculoskeletal } \\
\text { conditions, neurological } \\
\text { and cardiorespiratory } \\
\text { Cognitive: Healthy based } \\
\text { on cognitive test }\end{array}$ & $\begin{array}{l}\text { Total sample: NR; (I) NR (67.2 } \\
\pm 9.9) y ;(I I) ~ N R(66.3 \pm 9.8) y\end{array}$ \\
\hline
\end{tabular}

Past-week Physical Activity Questionnaires

\begin{tabular}{|c|c|c|}
\hline \multirow[t]{3}{*}{$\begin{array}{l}\text { AAS } \\
\text { Past-7 days }\end{array}$} & $\begin{array}{l}\text { Brown, } \\
\text { Trost [58] }\end{array}$ & $\begin{array}{l}\text { Assessed the test-retest reliability } \\
\text { of activity status derived from four } \\
\text { physical activity measures }\end{array}$ \\
\hline & $\begin{array}{l}\text { Brown, } \\
\text { Bauman } \\
{[59]}\end{array}$ & $\begin{array}{l}\text { Compared the level of agreement } \\
\text { in prevalence estimates of the } \\
\text { proportion of the population that } \\
\text { is sufficiently active for health } \\
\text { benefit derived from four measures } \\
\text { that are in use in Australia and } \\
\text { elsewhere around the world }\end{array}$ \\
\hline & $\begin{array}{l}\text { Creamer, } \\
\text { Bowles [60] }\end{array}$ & $\begin{array}{l}\text { Determining computer-assisted ap- } \\
\text { proaches for surveillance of phys- } \\
\text { ical activity }\end{array}$ \\
\hline $\begin{array}{l}\text { AAS } \\
\text { (modified) } \\
\text { Past-7 days }\end{array}$ & $\begin{array}{l}\text { Fjeldsoe, } \\
\text { Winkler } \\
{[18]}\end{array}$ & $\begin{array}{l}\text { Determined the test-retest } \\
\text { reliability and criterion validity the } \\
\text { Adapted Active Australia Survey } \\
\text { and whether these properties } \\
\text { varied across participants' activity } \\
\text { levels }\end{array}$ \\
\hline \multirow[t]{3}{*}{$\begin{array}{l}\text { CAQ-PAI } \\
\text { Past-7 days }\end{array}$} & $\begin{array}{l}\text { Mahabir, } \\
\text { Baer [61] }\end{array}$ & $\begin{array}{l}\text { Convergent validity of four } \\
\text { physical activity questionnaires } \\
\text { with DLW }\end{array}$ \\
\hline & $\begin{array}{l}\text { Rauh, } \\
\text { Hovell [62] }\end{array}$ & $\begin{array}{l}\text { Reliability and convergent validity } \\
\text { of several PAQs }\end{array}$ \\
\hline & $\begin{array}{l}\text { Washburn, } \\
\text { Smith [63] }\end{array}$ & Reliability of the CAQ-PAI \\
\hline
\end{tabular}

\begin{tabular}{|c|c|c|}
\hline $\begin{array}{l}\text { Checklist } \\
\text { Questionnaire } \\
\text { Past-7 days }\end{array}$ & $\begin{array}{l}\text { Masse, } \\
\text { Fulton [64] }\end{array}$ & $\begin{array}{l}\text { Compared the validity of two } \\
\text { physical activity questionnaire } \\
\text { formats }\end{array}$ \\
\hline $\begin{array}{l}\text { Global } \\
\text { Questionnaire } \\
\text { Past-7 days }\end{array}$ & $\begin{array}{l}\text { Masse, } \\
\text { Fulton [64] }\end{array}$ & As per Checklist Questionnaire \\
\hline $\begin{array}{c}\text { IPAQ-LF } \\
\text { Past-7 days }\end{array}$ & $\begin{array}{l}\text { Ahn, } \\
\text { Chmiel [65] }\end{array}$ & $\begin{array}{l}\text { Validity of IPAQ-SF (telephone) } \\
\text { with accelerometer amongst adults } \\
\text { with systemic lumpus } \\
\text { erythematosus }\end{array}$ \\
\hline
\end{tabular}

Garriguet, Validity of IPAQ-LF (self-adminisTremblay tered) and the new Physical Activ[66] ity for Adults Questionnaire (PAAQ) with accelerometers

IPAQ-SF Ainsworth, Compared the physical activity Past-7 days Macera prevalence estimates obtained [67] from BRFSS and IPAQ-SF (interview)

Brown, As for AAS Trost [58]
AAS (I), IPAQ (II), BRFSS

(III) \& NHS (IV):

Repeatability $(N=356$,

$104,127 \& 122)$

AAS (I), IPAQ (II) \&

BRFSS (III): Validity (N

$=428,427 \& 425)$

Validity \&

Repeatability $(\mathrm{N}=56)$

Validity \&

Repeatability $(\mathrm{N}=63)$

Physical: NR

Cognitive: Not screened

Total sample: $R=18-75 y(N R) y$; (I), (II), (III) \& (IV) 18-75y (NR)y

Physical: NR

Cognitive: Not screened

Total sample: $R=18-75 y$ (NR)y; (I), (II) \& (III) 18-75y (NR)y

Physical: NR

Total sample: NR $(43.1 \pm 11.4) y$

Cognitive: Screened

based on capability to read

Physical: NR

Cognitive: Screened based on capability to read

Validity $(N=65)$

Validity $(N=45)$

Combined gender (I), Male (II) \& Female (III): Repeatability $(N=633$, $261 \& 372)$

Validity $(N=260)$

Physical: NR

Physical: No chronic disease conditions Cognitive: Not screened

Physical: NR

Cognitive: Not screened

Physical: No chronic

disease conditions

Cognitive: Not screened

Cognitive: Not screened

As per Checklist

As per Checklist

Questionnaire

Physical: Systemic Lupus

Erythematosus

Cognitive: Not screened

IPAQ-LF (I) \& PAAQ (II): Physical: NR

Cognitive: Healthy based on cognitive interview

Validity $(N=94 \& 108)$

Validity $(N=9945)$

Physical: Noninstitutionalised

Cognitive: Noninstitutionalised

As for AAS
Total sample: 18-79 (NR); (I)

Total sample: 49.2-78.8 (59.9 $\pm 7.5) y$

Total sample: 18-55 (33.0 \pm 10.6)y

(I) Total sample: 25-65: (39.5

$\pm 10.8) y$; (II) NR (38.2 \pm 10.6$) y$;

(III) NR (40.5 \pm 10.8$)$

Total sample: $\mathrm{R}=40-70(49.2$

$\pm 7.0) y$

As per Checklist

Questionnaire

Total sample: NR $(45.4 \pm 10.9) \mathrm{y}$ NR $(47 \pm N R) y$; (II) NR $(47 \pm$ NR)y

Total sample: $\mathrm{R}=18-55+(\mathrm{NR})$ 
Table 5 Description of studies for the development and validation of interview-administered Past-week and Usual-week physical activity questionnaires (Continued)

\begin{tabular}{|c|c|c|c|c|c|}
\hline Instrument & Reference & Purpose of study & Study population & Health condition & $\begin{array}{l}\text { Age range (R; mean } \pm \\
\text { standard deviation) }\end{array}$ \\
\hline & $\begin{array}{l}\text { Brown, } \\
\text { Bauman } \\
{[59]}\end{array}$ & As for AAS & As for AAS & As for AAS & As for AAS \\
\hline $\begin{array}{l}\text { NZPAQ-LF } \\
\text { Past-7 days }\end{array}$ & $\begin{array}{l}\text { Moy, } \\
\text { Scragg [68] }\end{array}$ & $\begin{array}{l}\text { Convergent validity of NZPAQ-LF } \\
\text { with heart-rate monitoring }\end{array}$ & $\begin{array}{l}\text { Male (I) \& Female (II): } \\
\text { Validity ( } N=90 \text { \& } 96)\end{array}$ & $\begin{array}{l}\text { Physical: NR } \\
\text { Cognitive: Not screened }\end{array}$ & $\begin{array}{l}\text { Total sample: } 19-86(48.6 \pm \\
16.4) y ;(I) \text { NR }(48.4 \pm N R) y ;(I I) \\
\text { NR (48.7 } \pm \text { NR)y }\end{array}$ \\
\hline $\begin{array}{l}\text { NZPAQ-SF } \\
\text { Past-7 days }\end{array}$ & $\begin{array}{l}\text { Moy, } \\
\text { Scragg [68] }\end{array}$ & As per NZPAQ-LF & As per NZPAQ-LF & As per NZPAQ-LF & As per NZPAQ-LF \\
\hline $\begin{array}{c}\text { PAAQ } \\
\text { Past-7 days }\end{array}$ & $\begin{array}{l}\text { Garriguet, } \\
\text { Tremblay } \\
{[66]}\end{array}$ & As for IPAQ-LF & As for IPAQ-LF & As for IPAQ-LF & As for IPAQ-LF \\
\hline \multirow[t]{5}{*}{$\begin{array}{c}\text { PASE } \\
\text { Past-7 days }\end{array}$} & $\begin{array}{l}\text { Colbert, } \\
\text { Matthews } \\
{[52]}\end{array}$ & As for YPAS & As for YPAS & As for YPAS & As for YPAS \\
\hline & $\begin{array}{l}\text { Dinger, } \\
\text { Oman [69] }\end{array}$ & $\begin{array}{l}\text { Convergent validity and reliability } \\
\text { of PASE with accelerometers with } \\
\text { elderly individuals }\end{array}$ & $\begin{array}{l}\text { Validity \& } \\
\text { Repeatability }(N=56)\end{array}$ & $\begin{array}{l}\text { Physical: NR } \\
\text { Cognitive: Not screened }\end{array}$ & Total sample: NR $(75.7 \pm 7.9) y$ \\
\hline & $\begin{array}{l}\text { Johansen, } \\
\text { Painter [70] }\end{array}$ & $\begin{array}{l}\text { Convergent validity of three } \\
\text { physical activity questionnaires } \\
\text { with accelerometers in patients } \\
\text { with end-stage renal disease }\end{array}$ & Validity $(N=39)$ & $\begin{array}{l}\text { Physical: Patients } \\
\text { undergoing } \\
\text { haemodialysis } \\
\text { Cognitive: Not screened }\end{array}$ & Total sample: NR $(52 \pm 16) y$ \\
\hline & $\begin{array}{l}\text { Moore, Ellis } \\
{[57]}\end{array}$ & As for YPAS & As for YPAS & As for YPAS & As for YPAS \\
\hline & $\begin{array}{l}\text { Washburn, } \\
\text { Smith [71] }\end{array}$ & $\begin{array}{l}\text { Convergent validity and reliability } \\
\text { of PASE with accelerometers }\end{array}$ & $\begin{array}{l}\text { Validity \& } \\
\text { Repeatability }(N=119)\end{array}$ & $\begin{array}{l}\text { Physical: Included } \\
\text { participants without } \\
\text { serious physical } \\
\text { impairments } \\
\text { Cognitive: Included } \\
\text { participants without } \\
\text { serious cognitive } \\
\text { impairments, but } \\
\text { screening method not } \\
\text { clear }\end{array}$ & Total sample: NR $(73.4 \pm \mathrm{NR}) \mathrm{y}$ \\
\hline \multirow[t]{2}{*}{$\begin{array}{r}\text { PWMAQ } \\
\text { Past-7 days }\end{array}$} & $\begin{array}{l}\text { Pettee } \\
\text { Gabriel, } \\
\text { McClain } \\
\text { [72] }\end{array}$ & $\begin{array}{l}\text { Reliability and validity of PWMAQ } \\
\text { in middle-aged women }\end{array}$ & $\begin{array}{l}\text { Validity \& } \\
\text { Repeatability }(N=66)\end{array}$ & $\begin{array}{l}\text { Physical: NR } \\
\text { Cognitive: Not screened }\end{array}$ & Total sample: NR $(52.6 \pm 5.4) y$ \\
\hline & $\begin{array}{l}\text { Pettee } \\
\text { Gabriel, } \\
\text { McClain } \\
{[47]}\end{array}$ & As for MAQ & As for MAQ & As for MAQ & As for MAQ \\
\hline \multirow[t]{6}{*}{$\begin{array}{c}\text { PAR } \\
\text { Past-7 days }\end{array}$} & $\begin{array}{l}\text { Albanes, } \\
\text { Conway } \\
{[73]}\end{array}$ & As for CAQ-PAl & As for CAQ-PAI & As for CAQ-PAI & As for CAQ-PAl \\
\hline & $\begin{array}{l}\text { Blair, } \\
\text { Haskell [74] }\end{array}$ & Construct validity of PAR & $\begin{array}{l}\text { Male (I) \& Female (II): } \\
\text { Validity ( } N=1077, \\
\text { 1206) }\end{array}$ & $\begin{array}{l}\text { Physical: NR } \\
\text { Cognitive: Not screened }\end{array}$ & Total sample: 16-74 (NR)y \\
\hline & $\begin{array}{l}\text { Conway, } \\
\text { Seale [75] }\end{array}$ & $\begin{array}{l}\text { Convergent validity of PAR with } \\
\text { DLW }\end{array}$ & Validity $(N=24)$ & $\begin{array}{l}\text { Physical: No chronic } \\
\text { disease conditions } \\
\text { Cognitive: Not screened }\end{array}$ & $\begin{array}{l}\text { Total sample: } 27-65(41.2 \pm \\
\text { 2.0)y }\end{array}$ \\
\hline & $\begin{array}{l}\text { Garfield, } \\
\text { Canavan } \\
{[76]}\end{array}$ & As for PASE & As for PASE & As for PASE & As for PASE \\
\hline & $\begin{array}{l}\text { Gross, Sallis } \\
{[77]}\end{array}$ & Inter-rater reliability of PAR & $\begin{array}{l}\text { Inter-rater reliability } \\
(\mathrm{N}=21)\end{array}$ & $\begin{array}{l}\text { Physical: NR } \\
\text { Cognitive: Not screened }\end{array}$ & Total sample: 19-52 (NR)y \\
\hline & $\begin{array}{l}\text { Irwin, } \\
\text { Ainsworth }\end{array}$ & $\begin{array}{l}\text { Convergent validity of PAR with } \\
\text { DLW }\end{array}$ & Validity $(N=24)$ & $\begin{array}{l}\text { Physical: No chronic } \\
\text { disease conditions }\end{array}$ & $\begin{array}{l}\text { Total sample: } 27-65(41.2 \pm \\
\text { 9.6)y }\end{array}$ \\
\hline
\end{tabular}


Table 5 Description of studies for the development and validation of interview-administered Past-week and Usual-week physical activity questionnaires (Continued)

\begin{tabular}{|c|c|c|c|c|c|}
\hline Instrument & Reference & Purpose of study & Study population & Health condition & $\begin{array}{l}\text { Age range }(R ; \text { mean } \pm \\
\text { standard deviation) }\end{array}$ \\
\hline & {$[78]$} & & & Cognitive: Not screened & \\
\hline & $\begin{array}{l}\text { Johansen, } \\
\text { Painter [70] }\end{array}$ & As for PASE & As for PASE & As for PASE & As for PASE \\
\hline & $\begin{array}{l}\text { Mahabir, } \\
\text { Baer [61] }\end{array}$ & As for CAQ-PAI & As for CAQ-PAI & As for CAQ-PAl & As for CAQ-PAI \\
\hline & $\begin{array}{l}\text { Rauh, } \\
\text { Hovell [62] }\end{array}$ & As for CAQ-PAl & As for CAQ-PAl & As for CAQ-PAl & As for CAQ-PAl \\
\hline & $\begin{array}{l}\text { Sallis, } \\
\text { Haskell [79] }\end{array}$ & Reliability of PAR & Repeatability $(N=64)$ & $\begin{array}{l}\text { Physical: NR } \\
\text { Cognitive: Not screened }\end{array}$ & $\begin{array}{l}\text { Total sample: } 20-74(40.1 \pm \\
\text { 15.7)y }\end{array}$ \\
\hline & $\begin{array}{l}\text { Sarkin, } \\
\text { Johnson } \\
\text { [33] }\end{array}$ & $\begin{array}{l}\text { Construct validity of three physical } \\
\text { activity questionnaires }\end{array}$ & $\begin{array}{l}\text { Combined gender (I), } \\
\text { Male (II) \& Female (III): } \\
\text { Validity (N=575, } 256 \\
\text { \& 319) }\end{array}$ & $\begin{array}{l}\text { Physical: NR } \\
\text { Cognitive: Not screened }\end{array}$ & $\begin{array}{l}\text { (I) Total sample: NR }(24.5 \pm \\
1.9) y ;(I I) N R(24.7 \pm 2.0) y ; \text { (III) } \\
\text { NR (24.4 } \pm 2.1) y\end{array}$ \\
\hline & $\begin{array}{l}\text { Taylor, } \\
\text { Coffey [80] }\end{array}$ & $\begin{array}{l}\text { Convergent validity of PAR with } \\
\text { motion sensors }\end{array}$ & Validity $(N=30)$ & $\begin{array}{l}\text { Physical: Some patients } \\
\text { with myocardial } \\
\text { infarction several 11-26 } \\
\text { weeks prior to study } \\
\text { Cognitive: Not screened }\end{array}$ & $\begin{array}{l}\text { Total sample: 34-69 (52.3 } \pm \\
\text { NR) }\end{array}$ \\
\hline & $\begin{array}{l}\text { Washburn, } \\
\text { Jacobsen } \\
{[81]}\end{array}$ & $\begin{array}{l}\text { Convergent validity of PAR with } \\
\text { DLW }\end{array}$ & $\begin{array}{l}\text { Male (I) \& Female (II): } \\
\text { Validity }(N=17 \& 29)\end{array}$ & $\begin{array}{l}\text { Physical: No chronic } \\
\text { disease conditions } \\
\text { Cognitive: Not screened }\end{array}$ & $\begin{array}{l}\text { Total sample: } 17-35(23.6 \pm \\
4.2) y ; \text { (I) NR (23.9 } \pm 3.8) y \text {; (II) } \\
\text { NR (23.3 } \pm 4.6) y\end{array}$ \\
\hline & $\begin{array}{l}\text { Williams, } \\
\text { Klesges } \\
\text { [82] }\end{array}$ & $\begin{array}{l}\text { Reliability and convergent validity } \\
\text { of PAR in college students }\end{array}$ & $\begin{array}{l}\text { Repeatability \& } \\
\text { Validity }(\mathrm{N}=45)\end{array}$ & $\begin{array}{l}\text { Physical: NR } \\
\text { Cognitive: Not screened, } \\
\text { but were all enrolled at } \\
\text { a university }\end{array}$ & $\begin{array}{l}\text { Total sample: } 18-52(24.7 \pm \\
7.73) y\end{array}$ \\
\hline $\begin{array}{l}\text { VAPAQ } \\
\text { Past-7 days }\end{array}$ & $\begin{array}{l}\text { Betz, Myers } \\
{[83]}\end{array}$ & $\begin{array}{l}\text { Reproducibility of VAPAQ in an } \\
\text { elderly population }\end{array}$ & $\begin{array}{l}\text { Exercise group (I) \& } \\
\text { Usual care group (II): } \\
\text { Repeatability }(N=26 \\
\& 29) y\end{array}$ & $\begin{array}{l}\text { Physical: All patients had } \\
\text { abdominal aortic } \\
\text { aneurysm } \\
\text { Cognitive: Not screened }\end{array}$ & $\begin{array}{l}\text { Total sample: NR }(73.0 \pm 7.9) y \text {; } \\
\text { (I) NR; (II) NR }\end{array}$ \\
\hline
\end{tabular}

CaMos Canadian Multicentre Osteoporosis Study, IPEQ-WA Incidental and Planned Exercise Questionnaire, MAQ Modified Activity Questionnaire, NHS // Nurses' Health Study version II, Phone-FITT Phone Fitness, YPAS Yale Physical Activity Survey, AAS Active Australia Survey, CAQ-PAI College Alumni Questionnaire - Physical Activity Index, IPAQ-LF International Physical Activity Questionnaire Long Form, IPAQ-SF International Physical Activity Questionnaire Short Form, NZPAQ-LF New Zealand Physical Activity Questionnaire Long Form, NZPAQ-SF New Zealand Physical Activity Questionnaire Short Form, PAAQ Physical Activity Adult Questionnaire, PASE Physical Activity Scale for the Elderly, PWMAQ Past Week Modified Activity Questionnaire, PAR Physical Activity Recall Questionnaire, VAPAQ Veterans Physical Activity Questionnaire

respect to measurement type for each recall of PAQ, the convergent validity for direct measures had moderate correlations for both Usual-week $(r=0.33)$ and Past-week PAQs $(r=0.40)$ compared to weak correlations for indirect measures $(r=0.28$ and $r=0.19$, respectively). When Usual-week and Past-week PAQs were compared separately between direct and indirect measures, similar correlations were observed for the Past-week PAQs $(r=0.40)$ and the Usual-week PAQs $(r=0.33)$ for direct measures with moderate correlations. However, for indirect measures, there was a moderate correlation $(r=0.33)$ for Usual-week PAQs whereas the Past-week PAQs had a weak correlation $(r=0.19)$. Finally, when both Past-week and Usual-week PAQs were combined, direct measures had a moderate correlation $(r=0.39)$ whereas indirect measures had a weak correlation $(r=0.21)$.

Table 8 displays the quality of psychometric properties of both types of PAQs (i.e., Usual-week and Past-week) according to the criteria established by Terwee, Bot [15] and Cordier, Chen [30]. Table 9 provides the overall summary rating of the psychometric properties for each PAQ based on the levels of evidence by Schellingerhout, Verhagen [14]. According to Table 9, very few psychometric properties were reported (40 out of 120 possible ratings: $33.3 \%$ ), with the quality of psychometric properties primarily reported for reliability (13/20: 65\%) and hypothesis testing (18/20: 90\%). Fewer results were identified for internal consistency (2/20: $10 \%)$, content validity (3/20: 15\%) and measurement error (4/20: 20\%), while structural validity was not rated for any of the PAQs. Of all the psychometric properties rated for psychometric quality [32], the results were mainly negative (17/40: 42.5\%), consisting of "strong negative" (10/40: 25\%), "moderate negative" (5/40: 12.5\%) and "limited negative" (2/40: $5 \%)$. Several psychometric properties were reported with "conflicting" (13/40: 32.5\%), whilst 
Table 6 Overview of the methodological quality assessment of studies reporting on psychometric properties of interviewadministered Usual-week and Past-week physical activity questionnaires using the COSMIN checklist

\begin{tabular}{|c|c|c|c|c|c|c|c|}
\hline \multirow[t]{3}{*}{ Instrument } & \multirow[t]{3}{*}{ Study } & \multicolumn{6}{|c|}{ Measurement properties } \\
\hline & & \multirow{2}{*}{$\begin{array}{l}\text { Internal } \\
\text { consistency }\end{array}$} & \multirow[t]{2}{*}{ Reliability } & \multirow{2}{*}{$\begin{array}{l}\text { Measurement } \\
\text { error }\end{array}$} & \multirow{2}{*}{$\begin{array}{l}\text { Content } \\
\text { validity }\end{array}$} & \multirow{2}{*}{$\begin{array}{l}\text { Structural } \\
\text { validity }\end{array}$} & Hypothesis testing $^{\mathrm{ab}}$ \\
\hline & & & & & & & Type: Score \\
\hline \multicolumn{8}{|c|}{ Usual-week Physical Activity Questionnaires } \\
\hline $\begin{array}{c}\text { CaMos } \\
\text { Usual-week }\end{array}$ & Nadalin, Bentvelsen [36] & NR & 78.1\% (Excellent) ${ }^{c}$ & NR & NR & NR & NR \\
\hline $\begin{array}{l}\text { IPEQ } \\
\text { Usual-week }\end{array}$ & Merom, Delbaere [37] & NR & NR & NR & NR & NR & $\begin{array}{l}\text { Direct: } 68.7 \% \text { (Good) } \\
\text { Indirect: } 43.5 \% \text { (Fair) }\end{array}$ \\
\hline \multirow[t]{4}{*}{$\begin{array}{c}\text { MAQ } \\
\text { Usual-week }\end{array}$} & Pettee Gabriel, McClain [38] & NR & $71.4 \%(\mathrm{Good})^{c}$ & NR & NR & NR & $\begin{array}{l}\text { Direct: } 69.6 \% \text { (Good) } \\
\text { Indirect: } 59.1 \% \text { (Good) }\end{array}$ \\
\hline & Kriska, Knowler [39] & NR & $62.1 \%(\mathrm{Good})^{c}$ & NR & NR & NR & Direct: $43.5 \%$ (Fair) \\
\hline & Kriska, Edelstein [40] & NR & NR & NR & NR & NR & Direct: $78.3 \%$ (Excellent) \\
\hline & Schulz, Harper [41] & NR & NR & NR & NR & NR & Direct: $34.8 \%$ (Fair) \\
\hline $\begin{array}{c}\text { NHS II } \\
\text { Usual-week }\end{array}$ & Pettee Gabriel, McClain [38] & NR & $71.4 \%(\mathrm{Good})^{\mathrm{c}}$ & NR & NR & NR & $\begin{array}{l}\text { Direct: } 69.6 \% \text { (Good) } \\
\text { Indirect: } 59.1 \% \text { (Good) }\end{array}$ \\
\hline $\begin{array}{l}\text { Phone-FITT } \\
\text { Usual-week }\end{array}$ & Gill, Jones [32] & NR & $62.1 \%(\mathrm{Good})^{c}$ & NR & $28.5 \%$ (Fair) & NR & $\begin{array}{l}\text { Direct: } 69.6 \% \text { (Good) } \\
\text { Indirect: } 68.1 \% \text { (Good) }\end{array}$ \\
\hline \multirow{6}{*}{$\begin{array}{c}\text { YPAS } \\
\text { Usual-week }\end{array}$} & Colbert, Matthews [42] & NR & $72.4 \%(\text { Good) })^{c}$ & 75.9\% (Excellent) & NR & NR & Direct: 82.4\% (Excellent) \\
\hline & Dipietro, Caspersen [43] & $21.7 \%$ (Poor) & 82.8\% (Excellent) ${ }^{\dagger}$ & $65.5 \%$ (Good) & NR & NR & Direct: 56.5\% (Good) \\
\hline & Gennuso, Matthews [44] & NR & $71.9 \%(\mathrm{Good})^{\mathrm{c}}$ & NR & NR & NR & Direct: $60.9 \%$ (Good) \\
\hline & Harada, Chiu [45] & NR & NR & NR & NR & NR & $\begin{array}{l}\text { Direct: } 68.1 \% \text { (Good) } \\
\text { Indirect: } 75.5 \% \text { (Excellent) }\end{array}$ \\
\hline & Kolbe-Alexander, Lambert [46] & NR & $56.3 \%(\mathrm{Good})^{c}$ & $58.6 \%$ (Good) & NR & NR & Direct: 67.4\% (Good) \\
\hline & Moore, Ellis [47] & NR & NR & NR & NR & NR & Indirect: $73.9 \%$ (Good) \\
\hline \multicolumn{8}{|c|}{ Past-week Physical Activity Questionnaires } \\
\hline \multirow{3}{*}{$\begin{array}{l}\text { AAS } \\
\text { Past-7 days }\end{array}$} & Brown, Bauman [49] & NR & 78.1\% (Excellent) ${ }^{c}$ & NR & NR & NR & NR \\
\hline & Brown, Trost [48] & NR & NR & NR & NR & NR & Direct: 60.9\% (Good) \\
\hline & Creamer, Bowles [50] & NR & $72.4 \%(\mathrm{Good})^{\mathrm{c}}$ & NR & NR & NR & Direct: 82.6\% (Excellent) \\
\hline $\begin{array}{l}\text { AAS (modified) } \\
\text { Past-7 days }\end{array}$ & Fjeldsoe, Winkler [12] & NR & $72.2 \%(\text { Good })^{c}$ & $72.4 \%$ (Good) & NR & NR & Direct: 78.3\% (Excellent) \\
\hline \multirow{3}{*}{$\begin{array}{l}\text { CAQ-PAl } \\
\text { Past-7 days }\end{array}$} & Mahabir, Baer [51] & NR & NR & NR & NR & NR & Direct: 54.3\% (Good) \\
\hline & Rauh, Hovell [52] & NR & $65.5 \%(\mathrm{Good})^{c}$ & NR & NR & NR & $\begin{array}{l}\text { Direct: 60.9\% (Good) } \\
\text { Indirect: 60.9\% (Good) }\end{array}$ \\
\hline & Washburn, Smith [53] & NR & $72.4 \%(\mathrm{Good})^{\mathrm{c}}$ & NR & NR & NR & Indirect: 65.2\% (Good) \\
\hline $\begin{array}{l}\text { Checklist Questionnaire } \\
\text { Past-7 days }\end{array}$ & Masse, Fulton [54] & NR & NR & NR & NR & NR & Direct: 69.6\% (Good) \\
\hline $\begin{array}{l}\text { Global Questionnaire } \\
\text { Past-7 days }\end{array}$ & Masse, Fulton [54] & NR & NR & NR & NR & NR & Direct: 69.6\% (Good) \\
\hline \multirow{2}{*}{$\begin{array}{c}\text { IPAQ-LF } \\
\text { Past-7 days }\end{array}$} & Ahn, Chmiel [55] & NR & NR & NR & NR & NR & Direct: 78.3\% (Excellent) \\
\hline & Garriguet, Tremblay [56] & NR & NR & NR & NR & NR & Direct: 69.6\% (Good) \\
\hline \multirow{3}{*}{$\begin{array}{c}\text { IPAQ-SF } \\
\text { Past-7 days }\end{array}$} & Ainsworth, Macera [57] & NR & NR & NR & NR & NR & Direct: 78.2\% (Excellent) \\
\hline & Brown, Bauman [49] & NR & 78.1\% (Excellent) ${ }^{c}$ & NR & NR & NR & NR \\
\hline & Brown, Trost [48] & NR & NR & NR & NR & NR & Direct: 60.9\% (Good) \\
\hline $\begin{array}{l}\text { NZPAQ-LF } \\
\text { Past-7 days }\end{array}$ & Moy, Scragg [58] & NR & NR & NR & NR & NR & Indirect: $82.6 \%$ (Excellent) \\
\hline $\begin{array}{l}\text { NZPAQ-SF } \\
\text { Past-7 days }\end{array}$ & Moy, Scragg [58] & NR & NR & NR & NR & NR & Indirect: $82.6 \%$ (Excellent) \\
\hline $\begin{array}{c}\text { PAAQ } \\
\text { Past-7 days }\end{array}$ & Garriguet, Tremblay [56] & NR & NR & NR & $57.1 \%$ (Good) & NR & Direct: 78.3\% (Excellent) \\
\hline \multirow{3}{*}{$\begin{array}{c}\text { PASE } \\
\text { Past }-7 \text { days }\end{array}$} & Colbert, Matthews [42] & NR & $72.4 \%(\mathrm{Good})^{c}$ & 75.9\% (Excellent) & NR & NR & Direct: 82.4\% (Excellent) \\
\hline & Dinger, Oman [59] & NR & $72.4 \%(\text { Good })^{c}$ & NR & NR & NR & Direct: 82.6\% (Excellent) \\
\hline & Johansen, Painter [60] & NR & NR & NR & NR & $N R$ & $\begin{array}{l}\text { Direct: } 69.6 \% \text { (Good) } \\
\text { Indirect: } 43.5 \% \text { (Fair) }\end{array}$ \\
\hline
\end{tabular}


Table 6 Overview of the methodological quality assessment of studies reporting on psychometric properties of interviewadministered Usual-week and Past-week physical activity questionnaires using the COSMIN checklist (Continued)

\begin{tabular}{|c|c|c|c|c|c|c|c|}
\hline \multirow[t]{3}{*}{ Instrument } & \multirow[t]{3}{*}{ Study } & \multicolumn{6}{|c|}{ Measurement properties } \\
\hline & & \multirow{2}{*}{$\begin{array}{l}\text { Internal } \\
\text { consistency }\end{array}$} & \multirow[t]{2}{*}{ Reliability } & \multirow{2}{*}{$\begin{array}{l}\text { Measurement } \\
\text { error }\end{array}$} & \multirow{2}{*}{$\begin{array}{l}\text { Content } \\
\text { validity }\end{array}$} & \multirow{2}{*}{$\begin{array}{l}\text { Structural } \\
\text { validity }\end{array}$} & \multirow{2}{*}{$\begin{array}{l}\text { Hypothesis testing } \\
\text { Type: Score }\end{array}$} \\
\hline & & & & & & & \\
\hline \multirow{4}{*}{$\begin{array}{r}\text { PWMAQ } \\
\text { Past-7 days }\end{array}$} & Moore, Ellis [47] & NR & NR & NR & NR & NR & Indirect: 73.9\% (Good) \\
\hline & Washburn, Smith [61] & $43.5 \%$ (Fair) & 82.8\% (Excellent) ${ }^{c}$ & NR & $71.4 \%$ (Good) & NR & Indirect: 68.8\% (Good) \\
\hline & Pettee Gabriel, McClain [62] & NR & $72.4 \%(\text { Good })^{c}$ & NR & NR & NR & Direct: 69.6\% (Good) \\
\hline & Pettee Gabriel, McClain [38] & NR & $71.4 \%(\mathrm{Good})^{\mathrm{C}}$ & NR & NR & NR & $\begin{array}{l}\text { Direct: 69.6\% (Good) } \\
\text { Indirect: 59.1\% (Good) }\end{array}$ \\
\hline \multirow{14}{*}{$\begin{array}{c}\text { PAR } \\
\text { Past-7 days }\end{array}$} & Albanes, Conway [63] & NR & $N R$ & NR & NR & NR & Direct: 30.4\% (Fair) \\
\hline & Blair, Haskell [64] & NR & NR & NR & NR & NR & $\begin{array}{l}\text { Direct: } 78.3 \% \text { (Excellent) } \\
\text { Indirect: } 78.3 \% \text { (Excellent) }\end{array}$ \\
\hline & Conway, Seale [65] & NR & NR & NR & NR & NR & Direct: 69.6\% (Good) \\
\hline & Garfield, Canavan [66] & NR & NR & NR & NR & NR & Direct: 56.5\% (Good) \\
\hline & Gross, Sallis [67] & NR & $58.6 \%(G o o d)^{d}$ & NR & NR & NR & NR \\
\hline & Irwin, Ainsworth [68] & NR & NR & NR & NR & NR & $\begin{array}{l}\text { Direct: 52.2\% (Good) } \\
\text { Indirect: } 52.2 \% \text { (Good) }\end{array}$ \\
\hline & Johansen, Painter [60] & NR & NR & NR & NR & NR & $\begin{array}{l}\text { Direct: 65.2\% (Good) } \\
\text { Indirect) 56.5\% (Good) }\end{array}$ \\
\hline & Mahabir, Baer [51] & NR & NR & NR & NR & NR & Direct: 65.2\% (Good) \\
\hline & Rauh, Hovell [52] & NR & $65.5 \%(G o o d)^{c}$ & NR & NR & NR & $\begin{array}{l}\text { Direct: 60.9\% (Good) } \\
\text { Indirect: } 60.9 \% \text { (Good) }\end{array}$ \\
\hline & Sallis, Haskell [69] & $36.4 \%$ (Fair) & 79.3\% (Excellent) ${ }^{c}$ & NR & NR & NR & Indirect: 73.9\% (Good) \\
\hline & Sarkin, Johnson [70] & NR & NR & NR & NR & NR & Divergent: 34.8\% (Fair) \\
\hline & Taylor, Coffey [71] & NR & $N R$ & NR & NR & NR & $\begin{array}{l}\text { Direct: 56.5\% (Good) } \\
\text { Indirect: } 56.5 \% \text { (Good) }\end{array}$ \\
\hline & Washburn, Jacobsen [72] & NR & NR & NR & NR & NR & Direct: 65.2\% (Good) \\
\hline & Williams, Klesges [73] & NR & $55.2 \%(\mathrm{Good})^{c}$ & NR & NR & NR & Direct: 60.9\% (Good) \\
\hline $\begin{array}{c}\text { VAPAQ } \\
\text { Past-7 days }\end{array}$ & Betz, Myers [74] & NR & $58.6 \%(\mathrm{Good})^{c}$ & $62.1 \%$ (Good) & NR & NR & NR \\
\hline
\end{tabular}

${ }^{\mathrm{a}}$ Direct comparisons of physical activity measures (e.g. physical activity level between PAQ and other PAQs, diaries or objective measures)

${ }^{b}$ Indirect comparisons of physical activity measures (e.g. physical activity level between PAQ and physical fitness, given the assumption that individuals with greater level of physical activity would have a greater level of physical fitness)

'Test-retest reliability

dinter-rater reliability

fewer psychometric properties were reported for "indeterminate" (5/40: $12.5 \%)$. One psychometric property had a "not evaluated" rating, due to poor COSMIN scoring.

The relative number of negative ("strong negative" [6/ 18: 33.3\%] and "moderate negative" [3/18: 16.7\%]) and conflicting (8/18: 44.4\%) ratings were reported the most for hypothesis testing; only one "strong positive" rating was identified. For reliability, a greater relative number of positive ratings ("strong positive" [1/13: 7.7\%] and "moderate positive" [2/13: 15.4\%]) were found. However, reliability also exhibited several negative ("strong negative" [4/13: 30.8\%] and "moderate negative" [1/13: 7.7\%]) and conflicting (5/13: 38.5\%) ratings. The relative number of "indeterminate" ratings was greatest for measurement error (4/4: 100\%), whilst internal consistency showed only one "indeterminate", "limited negative" and "not evaluated" ratings (1/3: 33.3\%, respectively). There was one "moderate positive" rating (1/3: 33.3\%) for content validity, although the rest of the ratings consisted of one "moderate negative" and "limited negative" ratings (1/3: $33.3 \%$, respectively).

When comparing the PAQs, reliability demonstrated positive ratings for Past-Week Modifiable Activity Questionnaire (PWMAQ) ("strong positive"), Phone-FITT ("moderate positive") and the Veterans Physical Activity Questionnaire (VAPAQ) ("moderate positive"). However, the results for PWMAQ also demonstrated a "conflicting" rating in hypothesis testing, Phone-FITT received a "limited negative" and "moderate negative" in content validity and hypothesis testing, respectively, and VAPAQ received an "indeterminate" rating for measurement error. The AAS (modified) demonstrated a "strong positive" rating for hypothesis testing, although this PAQ also received a "strong negative" and "indeterminate" rating for reliability and measurement error, respectively. While PWMAQ, Phone-FITT, VAPAQ and AAS 
Table 7 The weighted mean of the $r$-values for reliability testing and convergent validity of Past-week and Usual-week physical activity questionnaires

\begin{tabular}{|c|c|c|}
\hline Instrument & $r$-values & Sample (n) \\
\hline \multicolumn{3}{|l|}{ Reliability testing } \\
\hline CaMos Usual-week & NR & NR \\
\hline IPEQ-WA Usual-week & NR & $N R$ \\
\hline MAQ Usual-week & 0.91 & 46 \\
\hline NHS II Usual 7-days & NR & $N R$ \\
\hline Phone-FITT Usual-week & NR & NR \\
\hline YPAS Usual 7-days & 0.56 & 198 \\
\hline AAS Past 7-days & NR & NR \\
\hline AAS (modified) Past 7-days & 0.65 & 63 \\
\hline CAQ-PAI Past 7-days & 0.53 & 633 \\
\hline Checklist Questionnaire Past 7-days & NR & NR \\
\hline Global Questionnaire Past 7-days & NR & $N R$ \\
\hline IPAQ-LF Past 7-days & NR & NR \\
\hline IPAQ-SF Past 7-days & NR & NR \\
\hline NZPAQ-LF Past 7-days & NR & $N R$ \\
\hline NZPAQ-SF Past 7-days & NR & NR \\
\hline PAAQ Past 7-days & NR & NR \\
\hline PASE Past 7-days & 0.68 & 144 \\
\hline PWMAQ Past 7-days & NR & NR \\
\hline PAR Past 7-days & 0.65 & 118 \\
\hline VAPAQ Past 7-days & 0.93 & 55 \\
\hline Average for Usual-week PAQs & 0.63 & 244 \\
\hline Average for Past-week PAQs & 0.56 & 950 \\
\hline \multicolumn{3}{|l|}{ Convergent validity testing } \\
\hline \multicolumn{3}{|l|}{ CaMos } \\
\hline \multicolumn{3}{|l|}{ Usual-week } \\
\hline Direct \& Indirect & NR & NR \\
\hline \multicolumn{3}{|l|}{ Direct } \\
\hline \multicolumn{3}{|l|}{ Indirect } \\
\hline \multicolumn{3}{|l|}{ IPEQ-WA } \\
\hline \multicolumn{3}{|l|}{ Usual-week } \\
\hline Direct \& Indirect & 0.28 & 553 \\
\hline Direct & 0.22 & 177 \\
\hline Indirect & 0.31 & 376 \\
\hline \multicolumn{3}{|l|}{ MAQ } \\
\hline \multicolumn{3}{|l|}{ Usual-week } \\
\hline Direct \& Indirect & 0.47 & 118 \\
\hline Direct & 0.57 & 118 \\
\hline Indirect & 0.23 & 66 \\
\hline \multicolumn{3}{|l|}{ NHS $\|$} \\
\hline \multicolumn{3}{|l|}{ Usual 7-days } \\
\hline Direct \& Indirect & 0.27 & 66 \\
\hline Direct & 0.43 & 66 \\
\hline
\end{tabular}


Table 7 The weighted mean of the $r$-values for reliability testing and convergent validity of Past-week and Usual-week physical activity questionnaires (Continued)

\begin{tabular}{|c|c|c|}
\hline Instrument & $r$-values & Sample (n) \\
\hline Indirect & 0.22 & 66 \\
\hline \multicolumn{3}{|l|}{ Phone-FITT } \\
\hline \multicolumn{3}{|l|}{ Usual-week } \\
\hline Direct \& Indirect & 0.36 & 84 \\
\hline Direct & 0.44 & 48 \\
\hline Indirect & 0.25 & 36 \\
\hline \multicolumn{3}{|l|}{ YPAS } \\
\hline \multicolumn{3}{|l|}{ Usual 7-days } \\
\hline Direct \& Indirect & 0.36 & 2099 \\
\hline Direct & 0.43 & 824 \\
\hline Indirect & 0.34 & 1182 \\
\hline \multicolumn{3}{|l|}{ AAS } \\
\hline \multicolumn{3}{|l|}{ Past 7-days } \\
\hline Direct \& Indirect & NR & NR \\
\hline Direct & $N R$ & NR \\
\hline Indirect & NR & NR \\
\hline \multicolumn{3}{|l|}{ AAS (modified) } \\
\hline \multicolumn{3}{|l|}{ Past 7-days } \\
\hline Direct \& Indirect & 0.57 & 63 \\
\hline Direct & 0.57 & 63 \\
\hline Indirect & NR & NR \\
\hline \multicolumn{3}{|l|}{ CAQ-PAI } \\
\hline \multicolumn{3}{|l|}{ Past 7-days } \\
\hline Direct \& Indirect & 0.15 & 3731 \\
\hline Direct & 0.46 & 65 \\
\hline Indirect & 0.14 & 3666 \\
\hline \multicolumn{3}{|l|}{ Checklist Questionnaire } \\
\hline \multicolumn{3}{|l|}{ Past 7-days } \\
\hline Direct \& Indirect & 0.46 & 2231 \\
\hline Direct & 0.46 & 2231 \\
\hline Indirect & $N R$ & NR \\
\hline \multicolumn{3}{|l|}{ Gobal Questionnaire } \\
\hline \multicolumn{3}{|l|}{ Past 7-days } \\
\hline Direct \& Indirect & 0.35 & 2231 \\
\hline Direct & 0.35 & 2231 \\
\hline Indirect & $N R$ & NR \\
\hline \multicolumn{3}{|l|}{ IPAQ-LF } \\
\hline \multicolumn{3}{|l|}{ Past 7-days } \\
\hline Direct \& Indirect & 0.23 & 436 \\
\hline Direct & 0.23 & 436 \\
\hline Indirect & NR & NR \\
\hline \multicolumn{3}{|l|}{ IPAQ-SF } \\
\hline Past 7-days & & \\
\hline
\end{tabular}


Table 7 The weighted mean of the $r$-values for reliability testing and convergent validity of Past-week and Usual-week physical activity questionnaires (Continued)

\begin{tabular}{|c|c|c|}
\hline Instrument & $r$-values & Sample (n) \\
\hline Direct \& Indirect & 0.34 & 25.2 \\
\hline Direct & 0.34 & 25.2 \\
\hline Indirect & NR & NR \\
\hline \multicolumn{3}{|l|}{ NZPAQ-LF } \\
\hline \multicolumn{3}{|l|}{ Past 7-days } \\
\hline Direct \& Indirect & NA & NA \\
\hline Direct & 0.25 & 186 \\
\hline Indirect & NR & NR \\
\hline \multicolumn{3}{|l|}{ NZPAQ-SF } \\
\hline \multicolumn{3}{|l|}{ Past 7-days } \\
\hline Direct \& Indirect & NR & NR \\
\hline Direct & 0.25 & 186 \\
\hline Indirect & NR & NR \\
\hline \multicolumn{3}{|l|}{ PAAQ } \\
\hline \multicolumn{3}{|l|}{ Past 7-days } \\
\hline Direct \& Indirect & 0.41 & 318 \\
\hline Direct & 0.41 & 318 \\
\hline Indirect & NR & $N R$ \\
\hline \multicolumn{3}{|l|}{ PASE } \\
\hline \multicolumn{3}{|l|}{ Past 7-days } \\
\hline Direct \& Indirect & 0.31 & 355 \\
\hline Direct & 0.44 & 95 \\
\hline Indirect & 0.27 & 260 \\
\hline \multicolumn{3}{|l|}{ PWMAQ } \\
\hline \multicolumn{3}{|l|}{ Past 7-days } \\
\hline Direct \& Indirect & 0.51 & 64 \\
\hline Direct & 0.51 & 64 \\
\hline Indirect & NR & NR \\
\hline \multicolumn{3}{|l|}{ PAR } \\
\hline \multicolumn{3}{|l|}{ Past 7-days } \\
\hline Direct \& Indirect & 0.25 & 3539 \\
\hline Direct & 0.39 & 874 \\
\hline Indirect & 0.21 & 2547 \\
\hline \multicolumn{3}{|l|}{ VAPAQ } \\
\hline \multicolumn{3}{|l|}{ Past 7-days } \\
\hline Direct \& Indirect & NR & NR \\
\hline Direct & NR & NR \\
\hline Indirect & NR & NR \\
\hline \multirow[t]{3}{*}{ Average for Usual-week PAQs } & Direct \& Indirect $(r=0.30)$ & Direct \& Indirect $(n=4730)$ \\
\hline & Direct $(r=0.33)$ & Direct $(n=2019)$ \\
\hline & Indirect $(r=0.28)$ & Indirect $(n=2711)$ \\
\hline \multirow[t]{2}{*}{ Average for Past-week PAQs } & Direct \& Indirect $(r=0.28)$ & Direct \& Indirect $(n=14,147)$ \\
\hline & Direct $(r=0.40)$ & Direct $(n=6182)$ \\
\hline
\end{tabular}


Table 7 The weighted mean of the $r$-values for reliability testing and convergent validity of Past-week and Usual-week physical activity questionnaires (Continued)

\begin{tabular}{lll}
\hline Instrument & $r$-values & Sample $(n)$ \\
\hline & Indirect $(r=0.19)$ & Indirect $(n=7965)$ \\
Past-week and Usual-week PAQs & Direct $(r=0.39)$ & Direct $(n=8201)$ \\
& Indirect $(r=0.21)$ & Indirect $(n=10,676)$
\end{tabular}

Notes. CaMos Canadian Multicentre Osteoporosis Study, IPEQ-WA Incidental and Planned Exercise Questionnaire, MAQ Modified Activity Questionnaire, NHS /I Nurses' Health Study version II, Phone-FITT Phone Fitness, YPAS Yale Physical Activity Survey, AAS Active Australia Survey, CAQ-PAI College Alumni Questionnaire Physical Activity Index, IPAQ-LF International Physical Activity Questionnaire Long Form, IPAQ-SF International Physical Activity Questionnaire Short Form, NZPAQLF New Zealand Physical Activity Questionnaire Long Form, NZPAQ-SF New Zealand Physical Activity Questionnaire Short Form, PAAQ Physical Activity Adult Questionnaire, PASE Physical Activity Scale for the Elderly, PWMAQ Past Week Modified Activity Questionnaire PAR Physical Activity Recall Questionnaire, VAPAQ Veterans Physical Activity Questionnaire

(modified) received mixed results, these measures have a substantial number of psychometric properties that were not reported.

When compared between the types of PAQs, a similar percentage of negative ratings (limited, moderate or strong) were shown for Past-week (12/28: 42.9\%) and Usual-week (5/12: 41.7\%) PAQs. Conversely, the relative number of positive ratings (limited, moderate or strong) for the Past-week PAQs (4/28: 14.3\%) was greater than Usual-week PAQs (1/12: 8.3\%), although the absolute number of "positive" ratings were small. The number of NR ratings [33] were noticeable across all PAQs.

\section{Discussion}

This systematic review examined the methodological quality of studies that investigated the psychometric properties of interview-administered, Usual-week and Past-week PAQs, in an adult population. There were 20 PAQs with 42 corresponding articles that reported on the psychometric properties of PAQs, of which 6 were Usual-week and 14 were Past-week PAQs. Amongst the psychometric properties, hypothesis testing was reported most frequently, followed by reliability, whereas measurement error, content validity and internal consistency were the least reported. Furthermore, structural validity was not reported in any of the included studies. The methodological quality of the studies exhibited good to excellent ratings across most of the psychometric properties. As per the average weighted mean of the $r$-values, both Usual-week PAQs and Past-week PAQs showed moderate correlations for reliability and convergent validity for direct measures, whereas convergent validity for indirect measures exhibited weak correlations irrespective of the type of PAQ. When comparing the weighted mean of the $r$-values between PAQ types, convergent validity for direct measures indicated moderate correlations for both Past-week and Usual-week PAQs, although convergent validity for indirect measures demonstrated moderate correlations for Usual-week PAQs, while weak correlations were observed for Past-week PAQs. According to the level of evidence (i.e., overall quality), most of the psychometric properties exhibited "moderate negative" to "strong negative" ratings irrespective of PAQ types, highlighting concerns for utilising current interview-administered PAQs.

\section{Quality of studies based on the COSMIN taxonomy}

Of the psychometric property reliability, most psychometric studies reported on test-retest reliability with good to excellent COSMIN ratings, whereas measurement error was only reported for four measures (YPAS, AAS [modified], PASE and VAPAQ), also with good to excellent ratings. Measurement error is an essential property of the reliability dimension, as it quantifies the magnitude of systematic and random error of PA levels that is not caused by true changes in the construct being measured; thus allowing practitioners to establish meaningful differences in PA measures [34]. Subsequently, more research is warranted to determine the measurement error of PAQs when administered in an interview setting. Compared to test-retest reliability and measurement error, internal consistency was reported for only three measures (YPAS, PASE and PAR) with poor to fair ratings. This discrepancy was due to included studies consisting of a small sample size and/or examining correlations between different items of the same PAQ without conducting Cronbach alpha statistic and factor analyses. According to Terwee et al. [13], both statistical approaches determine whether all items measure the same construct and checks the uni-dimensionality of the scale. Thus, future studies should consider these limitations when examining the internal consistency of interview-administered PAQs.

With the exception of two PAQs (CaMos and VAPAQ), hypothesis testing was reported for all PAQs with the majority of included studies reporting good to excellent ratings for methodological quality. However, studies only investigated three PAQs (Phone-FITT, PAAQ and PASE) for content validity with fair to good COSMIN ratings. The methodological limitations identified from these studies included lack of description on whether piloting was conducted by investigators, expert practitioners and/or the target population. Thus, future studies should consider these issues when examining the 
Table 8 Quality of psychometric properties per study based on the criteria by Terwee, Bot [9] and Cordier, Chen [30]

\begin{tabular}{|c|c|c|c|c|c|c|c|}
\hline \multirow[t]{3}{*}{ Assessment } & \multirow[t]{3}{*}{ Reference } & \multicolumn{6}{|c|}{ Measurement properties of questionnaires } \\
\hline & & \multicolumn{4}{|l|}{ Reliability } & \multicolumn{2}{|c|}{ Construct validity } \\
\hline & & $\begin{array}{l}\text { Internal } \\
\text { Consistency }\end{array}$ & Reliability & $\begin{array}{l}\text { Measurement } \\
\text { error }\end{array}$ & $\begin{array}{l}\text { Content } \\
\text { validity }\end{array}$ & $\begin{array}{l}\text { Structural } \\
\text { validity }\end{array}$ & $\begin{array}{l}\text { Hypothesis } \\
\text { testing }\end{array}$ \\
\hline \multicolumn{8}{|c|}{ Usual-week Physical Activity Questionnaires } \\
\hline CaMos Usual-week & $\begin{array}{l}\text { Nadalin, Bentvelsen } \\
\text { [36] }\end{array}$ & NR & - & NR & NR & NR & NR \\
\hline IPEQ Usual-week & Merom, Delbaere [37] & NR & NR & NR & NR & NR & $\begin{array}{l}\text { - (Direct) } \\
\text { - (Indirect) }\end{array}$ \\
\hline \multirow[t]{4}{*}{ MAQ Usual-week } & $\begin{array}{l}\text { Pettee Gabriel, } \\
\text { McClain [38] }\end{array}$ & NR & - & $N R$ & NR & $N R$ & $\begin{array}{l}\text { - (Direct) } \\
\text { - (Indirect) }\end{array}$ \\
\hline & Kriska, Knowler [39] & NR & + & NR & NR & NR & \pm (Direct) \\
\hline & Kriska, Edelstein [40] & NR & NR & NR & NR & NR & - (Direct) \\
\hline & Schulz, Harper [41] & $N R$ & NR & NR & NR & $N R$ & $+($ Direct $)$ \\
\hline NHS II Usual-week & $\begin{array}{l}\text { Pettee Gabriel, } \\
\text { McClain [38] }\end{array}$ & NR & - & NR & NR & NR & $\begin{array}{l} \pm \text { (Direct) } \\
\text { - (Indirect) }\end{array}$ \\
\hline Phone-FITT Usual-week & Gill, Jones [32] & NR & + & NR & - & $N R$ & $\begin{array}{l}\text { - (Direct) } \\
\text { - (Indirect) }\end{array}$ \\
\hline \multirow[t]{6}{*}{ YPAS Usual-week } & Colbert, Matthews [42] & $N R$ & + & $?$ & NR & $N R$ & - (Direct) \\
\hline & $\begin{array}{l}\text { Dipietro, Caspersen } \\
\text { [43] }\end{array}$ & NE & - & $?$ & NR & $N R$ & $\begin{array}{l}\text { - (Direct) } \\
\text { - (Indirect) }\end{array}$ \\
\hline & $\begin{array}{l}\text { Gennuso, Matthews } \\
\text { [44] }\end{array}$ & NR & - & NR & NR & NR & $?$ (Direct) \\
\hline & Harada, Chiu [45] & NR & NR & NR & NR & NR & $\begin{array}{l}+ \text { (Direct) } \\
- \text { (Indirect) }\end{array}$ \\
\hline & $\begin{array}{l}\text { Kolbe-Alexander, } \\
\text { Lambert [46] }\end{array}$ & NR & - & $?$ & NR & NR & - (Direct) \\
\hline & Moore, Ellis [47] & NR & NR & NR & NR & NR & - (Indirect) \\
\hline \multicolumn{8}{|c|}{ Past-week Physical Activity Questionnaires } \\
\hline \multirow[t]{3}{*}{ Active Australia Survey Past week } & Brown, Bauman [49] & $N R$ & - & $N R$ & NR & $N R$ & NR \\
\hline & Brown, Trost [48] & NR & NR & NR & NR & NR & - (Direct) \\
\hline & Creamer, Bowles [50] & $N R$ & + & NR & NR & $N R$ & - (Direct) \\
\hline $\begin{array}{l}\text { Active Australia Survey } \\
\text { (modified) Past-week }\end{array}$ & Fjeldsoe, Winkler [12] & NR & - & $?$ & NR & $N R$ & $+($ Direct $)$ \\
\hline \multirow[t]{3}{*}{ CAQ-PAI Past-week } & Mahabir, Baer [51] & $N R$ & NR & NR & NR & $N R$ & - (Direct) \\
\hline & Rauh, Hovell [52] & NR & - & NR & NR & NR & $\begin{array}{l}\text { - (Direct) } \\
\text { - (Indirect) }\end{array}$ \\
\hline & Washburn, Smith [53] & NR & - & $N R$ & NR & $N R$ & $\begin{array}{l}- \text { (Indirect) } \\
+ \\
\text { (Discriminant) }\end{array}$ \\
\hline $\begin{array}{l}\text { Checklist Questionnaire Past- } \\
\text { week }\end{array}$ & Masse, Fulton [54] & NR & NR & $N R$ & NR & $N R$ & \pm (Direct) \\
\hline Global Questionnaire Past-week & Masse, Fulton [54] & NR & $N R$ & $N R$ & $N R$ & $N R$ & - (Direct) \\
\hline \multirow{2}{*}{$\begin{array}{l}\text { IPAQ-LF (self-administered) Past- } \\
\text { week }\end{array}$} & Ahn, Chmiel [55] & NR & NR & NR & NR & NR & - (Direct) \\
\hline & $\begin{array}{l}\text { Garriguet, Tremblay } \\
\text { [56] }\end{array}$ & $N R$ & $N R$ & $N R$ & NR & $N R$ & - (Direct) \\
\hline \multirow[t]{3}{*}{ IPAQ-SF(interview) Past-week } & $\begin{array}{l}\text { Ainsworth, Macera } \\
\text { [57] }\end{array}$ & $N R$ & NR & NR & NR & $N R$ & - (Direct) \\
\hline & Brown, Bauman [49] & $N R$ & - & NR & $N R$ & $N R$ & NR \\
\hline & Brown, Trost [48] & NR & NR & NR & NR & NR & - (Direct) \\
\hline NZPAQ-LF Past-week & Moy, Scragg [58] & $N R$ & NR & NR & NR & $N R$ & - (Direct) \\
\hline
\end{tabular}


Table 8 Quality of psychometric properties per study based on the criteria by Terwee, Bot [9] and Cordier, Chen [30] (Continued)

\begin{tabular}{|c|c|c|c|c|c|c|c|}
\hline \multirow[t]{3}{*}{ Assessment } & \multirow[t]{3}{*}{ Reference } & \multicolumn{6}{|c|}{ Measurement properties of questionnaires } \\
\hline & & \multicolumn{4}{|l|}{ Reliability } & \multicolumn{2}{|c|}{ Construct validity } \\
\hline & & $\begin{array}{l}\text { Internal } \\
\text { Consistency }\end{array}$ & Reliability & $\begin{array}{l}\text { Measurement } \\
\text { error }\end{array}$ & $\begin{array}{l}\text { Content } \\
\text { validity }\end{array}$ & $\begin{array}{l}\text { Structural } \\
\text { validity }\end{array}$ & $\begin{array}{l}\text { Hypothesis } \\
\text { testing }\end{array}$ \\
\hline NZPAQ-SF Past-week & Moy, Scragg [58] & NR & NR & NR & NR & NR & - (Direct) \\
\hline PAAQ Past-week & $\begin{array}{l}\text { Garriguet, Tremblay } \\
\text { [56] }\end{array}$ & NR & NR & $N R$ & - & NR & - (Direct) \\
\hline \multirow[t]{5}{*}{ PASE Past-week } & Colbert, Matthews [42] & NR & - & $?$ & NR & NR & - (Direct) \\
\hline & Dinger, Oman [59] & NR & + & NR & NR & NR & - (Direct) \\
\hline & Johansen, Painter [60] & NR & NR & NR & NR & NR & $\begin{array}{l}+ \text { (Direct) } \\
- \text { (Indirect) }\end{array}$ \\
\hline & Moore, Ellis [47] & NR & NR & NR & NR & $N R$ & - (Indirect) \\
\hline & Washburn, Smith [61] & - & - & NR & + & $N R$ & - (Indirect) \\
\hline \multirow[t]{2}{*}{ PWMAQ Past-week } & $\begin{array}{l}\text { Pettee Gabriel, } \\
\text { McClain [62] }\end{array}$ & NR & + & $N R$ & $N R$ & $N R$ & $+($ Direct $)$ \\
\hline & $\begin{array}{l}\text { Pettee Gabriel, } \\
\text { McClain [38] }\end{array}$ & NR & + & $N R$ & $N R$ & NR & $\begin{array}{l}\text { - (Direct) } \\
\text { - (Indirect) }\end{array}$ \\
\hline \multirow[t]{14}{*}{ PAR Past-week } & Albanes, Conway [63] & NR & $N R$ & $N R$ & NR & NR & - (Direct) \\
\hline & Blair, Haskell [64] & NR & $N R$ & NR & $N R$ & $N R$ & $\begin{array}{l}\text { - (Direct) } \\
\text { - (Indirect) }\end{array}$ \\
\hline & Conway, Seale [65] & NR & $N R$ & $N R$ & $N R$ & $N R$ & - (Direct) \\
\hline & Garfield, Canavan [66] & NR & $N R$ & $N R$ & NR & $N R$ & + (Direct) \\
\hline & Gross, Sallis [67] & NR & + & NR & $N R$ & $N R$ & $N R$ \\
\hline & Irwin, Ainsworth [68] & NR & $N R$ & $N R$ & NR & NR & - (Indirect) \\
\hline & Johansen, Painter [60] & NR & NR & $N R$ & $N R$ & $N R$ & $\begin{array}{l}+ \text { (Direct) } \\
- \text { (Indirect) }\end{array}$ \\
\hline & Mahabir, Baer [51] & NR & NR & $N R$ & $N R$ & $N R$ & - (Direct) \\
\hline & Rauh, Hovell [52] & NR & - & $N R$ & $N R$ & $N R$ & $\begin{array}{l}\text { - (Direct) } \\
\text { - (Indirect) }\end{array}$ \\
\hline & Sallis, Haskell [69] & $?$ & - & $N R$ & $N R$ & $N R$ & - (Indirect) \\
\hline & Sarkin, Johnson [70] & NR & NR & $N R$ & $N R$ & NR & ? (Indirect) \\
\hline & Taylor, Coffey [71] & NR & $N R$ & $N R$ & $N R$ & $N R$ & $+($ Direct $)$ \\
\hline & $\begin{array}{l}\text { Washburn, Jacobsen } \\
\text { [72] }\end{array}$ & NR & NR & $N R$ & NR & $N R$ & $\begin{array}{l}\text { - (Direct) } \\
\text { - (Indirect) }\end{array}$ \\
\hline & Williams, Klesges [73] & NR & + & $N R$ & $N R$ & $N R$ & \pm (Direct) \\
\hline VAPAQ Past-week & Betz, Myers [74] & NR & + & $?$ & $N R$ & $N R$ & $N R$ \\
\hline
\end{tabular}

Notes. ${ }^{a}$ Direct comparisons of physical activity measures (e.g., physical activity level between PAQ and other PAQs, diaries or objective measures)

${ }^{\mathrm{b}}$ Indirect comparisons of physical activity measures (e.g., physical activity level between PAQ and physical fitness, given the assumption that individuals with greater level of physical activity would have a greater level of physical fitness)

content validity of PAQs. Most alarming is that none of the PAQs investigated structural validity. This means that the underlying constructs of all the PAQs are currently unknown, as appropriate statistical analyses to ascertain the factor structure or dimensionality (e.g., dimensionality and principle component analysis using Rasch analysis and exploratory and/or confirmatory factor analysis) of the measures and associated subscales have not been conducted. Therefore, when assessing structural validity of PAQs, future studies should identify whether their approach is in accordance with a formative (i.e., integrative items forming a construct) or reflective (i.e., items are reflective of the same underlying constructs) model.

\section{Quality of psychometric properties}

In the current review, test-retest reliability for the Usual-week PAQs was comparable to the Past-week PAQs based on the average weighted mean of the $r$-values. These results are in contrast to a previous study by Delbaere, Hauer [35], who compared the reliability of self-administered incidental and planned exercise 
Table 9 Overall quality score of psychometric properties for each interview-administered Usual-week and Past-week physical activity questionnaire using the levels of evidence by Schellingerhout et al., [14]

\begin{tabular}{|c|c|c|c|c|c|c|}
\hline Assessment & $\begin{array}{l}\text { Internal } \\
\text { Consistency }\end{array}$ & Reliability & $\begin{array}{l}\text { Measurement } \\
\text { error }\end{array}$ & Content validity & $\begin{array}{l}\text { Structural } \\
\text { validity }\end{array}$ & $\begin{array}{l}\text { Hypothesis } \\
\text { testing }\end{array}$ \\
\hline CaMos Usual-week & NR & Strong Negative & NR & NR & NR & NR \\
\hline IPEQ Usual-week & NR & NR & NR & NR & NR & $\begin{array}{l}\text { Moderate } \\
\text { Negative }\end{array}$ \\
\hline MAQ Usual-week & NR & Conflicting & NR & $\mathrm{NE}$ & NR & Conflicting \\
\hline NHS II Usual-week & NR & $\begin{array}{l}\text { Moderate } \\
\text { Negative }\end{array}$ & NR & NR & NR & Conflicting \\
\hline Phone-FITT Usual-week & NR & $\begin{array}{l}\text { Moderate } \\
\text { Positive }\end{array}$ & NR & Limited Negative & NR & $\begin{array}{l}\text { Moderate } \\
\text { Negative }\end{array}$ \\
\hline YPAS Usual-week & Not Evaluated & Conflicting & Indeterminate & NR & NR & Conflicting \\
\hline AAS Past -7 days & NR & Conflicting & NR & NR & NR & Strong Negative \\
\hline AAS (modified) Past-7 days & NR & Strong Negative & Indeterminate & NR & NR & Strong Positive \\
\hline CAQ-PAI Past-7 days & NR & Strong Negative & NR & NR & NR & Conflicting \\
\hline Checklist Questionnaire Past-7 days & NR & NR & NR & NR & NR & Conflicting \\
\hline Global Questionnaire Past-7 days & NR & NR & NR & NR & NR & $\begin{array}{l}\text { Moderate } \\
\text { Negative }\end{array}$ \\
\hline $\begin{array}{l}\text { IPAQ-LF (self-administered) Past-7 } \\
\text { days }\end{array}$ & NR & NR & NR & NR & NR & Strong Negative \\
\hline IPAQ-SF (interview) Past-7 days & NR & Strong Negative & NR & NR & NR & Strong Negative \\
\hline NZPAQ-LF Past-7 days & NR & NR & NR & NR & NR & Strong Negative \\
\hline NZPAQ-SF Past-7 days & NR & NR & NR & NR & NR & Strong Negative \\
\hline PAAQ Past -7 days & NR & NR & NR & $\begin{array}{l}\text { Moderate } \\
\text { Negative }\end{array}$ & NR & Strong Negative \\
\hline PASE Past- 7 days & Limited Negative & Conflicting & Indeterminate & $\begin{array}{l}\text { Moderate } \\
\text { Positive }\end{array}$ & NR & Conflicting \\
\hline PWMAQ Past-7 days & NR & Strong Positive & NR & NR & NR & Conflicting \\
\hline PAR Past -7 days & Indeterminate & Conflicting & NR & NR & NR & Conflicting \\
\hline VAPAQ Past-7 days & NR & $\begin{array}{l}\text { Moderate } \\
\text { Positive }\end{array}$ & Indeterminate & NR & NR & NR \\
\hline
\end{tabular}

Notes. CaMos Canadian Multicentre Osteoporosis Study, IPEQ-WA Incidental and Planned Exercise Questionnaire, MAQ Modified Activity Questionnaire, NHS II Nurses' Health Study version II, Phone-FITT Phone Fitness, YPAS Yale Physical Activity Survey, AAS Active Australia Survey, CAQ-PAI - College Alumni Questionnaire - Physical Activity Index; IPAQ-LF International Physical Activity Questionnaire Long Form, IPAQ-SF International Physical Activity Questionnaire Short Form, NZPAQLF New Zealand Physical Activity Questionnaire Long Form, NZPAQ-SF New Zealand Physical Activity Questionnaire Short Form, PAAQ Physical Activity Adult Questionnaire, PASE Physical Activity Scale for the Elderly, PWMAQ Past Week Modified Activity Questionnaire, PAR Physical Activity Recall Questionnaire, VAPAQ Veterans Physical Activity Questionnaire

questionnaire (IPEQ) between Usual-week (i.e., past three months) and Past-week versions. According to their results, the Usual-week IPEQ $(I C C=0.84)$ version showed greater test-retest reliability compared to the Past-week IPEQ version $(I C C=0.77)$. Authors speculated that PAQs with usual 7-day recall periods during the past several months exhibit better stability in PA measures across time points, compared with PAQs with past-week recalls given that PA levels may fluctuate from week-to-week [10], or season-to-season [11]. The discrepancies in findings between the current review, and the work by Delbaere, Hauer [35], may be due to distinct acceptable cut-off levels being employed for test-retest reliability. For example, Delbaere, Hauer [35] established acceptable ICC values at $\geq 0.6$, whereas the current review utilised an acceptable ICC criteria of $\geq 0.7$ according to the criteria set out by [15]. Therefore, where ICC values $(\geq 0.6$ to $<0.7)$ were classified as "acceptable" for [35], would have been considered below the acceptable cut-off level in the current review with a "negative" rating. In addition, the test-retest reliability in the current review was compared between PAQs with different recall methods based on average weighted mean of the $r$-values across multiple studies, whereas [35] compared different recall versions of IPEQ within the same study and population. Subsequently, the variation in study design and the type of PAQs may have diluted potential differences in the weighted mean of the $r$-values between Past-week and Usual-week PAQs in the current review. This is further supported by a previous 
systematic review by [16], who also reported comparable test-retest reliability of average weighted mean of the $r$-values for self-administered Usual-week and Past-week PAQs.

When comparing convergent validity (i.e., hypothesis testing) between PAQ recall types, the average weighted mean of the $r$-values of Past-week PAQs were comparable with Usual-week PAQs for direct measures. However, the average weighted mean of the $r$-values was greater for Usual-week PAQs compared with Past-week PAQs within our review. These findings differ to a previous systematic review reported by [16] in self-reported PAQs where convergent validity for direct measures were greater for Past-week PAQs than Usual-week PAQs. In addition, the average weighted mean of the $r$-values for direct measures of convergent validity for both PAQ recall periods showed a moderate correlation $(r=0.35)$, whereas Doma, Speyer [16] reported weak correlations for the same measures $(r=0.27)$ based on self-reported PAQs in their previous review. This trend has also been reported by previous studies that compared convergent validity between interview-administered and self-administered PAQs [36, 37]. For example, Chu, Ng [36] reported stronger associations between Global Physical Activity Questionnaire (GPAQ) for the interview-administered method $(r=0.44-0.52)$ compared with the self-administered method $(r=0.28-0.38)$ when compared against accelerometers. Collectively, PAQs administered via interview may allow reporting of PA levels with greater accuracy than by self-administration, possibly due to minimisation of respondent bias [36].

While weighted-mean of the $r$-values for direct measures of convergent validity were comparable between Past-week and Usual-week PAQs, indirect measures of convergent validity were stronger for Usual-week PAQs (i.e., moderate correlations) than Past-week PAQs (i.e., weak correlations). This suggests that Usual-week PAQs better reflect physical fitness (e.g., $\mathrm{VO}_{2 \max }, 6$-min walk test) and its associated physiological conditions (e.g., BMI, body fat percentage) than Past-week PAQs when administered via interviews. These results are expected, given that physical fitness measures are stable across several weeks despite exercise termination [38], as opposed to the inherent week-to-week fluctuations observed with PA level [39]. Subsequently, when estimating physical fitness levels based on PA level ascertained from PAQs, we encourage the use of Usual-week PAQs rather than Past-week PAQs, particularly when administered via interviews. However, it should be noted that the current review included studies consisting of older adults with a number of pathological conditions (e.g., cardiovascular disease, musculoskeletal disease and neurological disease), where chronic exercise adaptations and deconditioning may differ in response to apparently healthy, younger individuals [32, 40, 41]. Separating these populations was difficult in the current review as the majority of studies incorporated apparently healthy participants with those who had several pathological conditions in the one study. Thus, future research should systematically compare psychometric properties of PAQs between individuals with pathological conditions and their apparently healthy counterparts.

For the overall Level of Evidence, irrespective of recall methods, there was a substantial number of missing psychometric data (i.e., not reported [NR]), indicating that the psychometric properties pertinent to determining the quality of current PAQs are not being examined effectively. Of the few psychometric properties reported, there were only four that reported "moderate positive" to "strong positive" ratings, with the rest as "strong to limited negative", "indeterminate" and "conflicting" ratings, which demonstrate the weaknesses of current PAQs. No studies examined structural validity of PAQs, and only three PAQs examined internal consistency with ratings of "not evaluated", "indeterminate" and "limited negative". These weak results and lack of reporting is particularly concerning given that both structural validity and internal consistency are based on a reflective model, whereby all items are manifestations of the same underlying construct [27]. In addition, only a very limited number of PAQs reported on content validity (3/20: 15\%), with one "positive" rating and two "negative" ratings. These findings further raise the limitations of current interview-administered PAQs, as content validity measures the degree to which the content of a PAQ is an adequate reflection of the construct being measured [27].

When comparing the overall psychometric qualities of PAQs based on Level of Evidence between recall methods, there were minute differences between Usual-week and Past-week PAQs, which are in line with findings by [16]. Additionally, the "moderate negative" to "strong negative" ratings shown for the majority of psychometric properties in the current review are similar to those reported by other systematic reviews [16, 42, 43]. These psychometric properties were rated poorly as the correlations were predominantly below the acceptable levels for test-retest reliability and convergent validity. However, authors from several studies included in the current review reported that the PAQs demonstrated acceptable test-retest reliability and convergent validity, which conflicts with findings from this current review. The discrepancy in these interpretations is because authors in the included studies considered test-retest reliability and convergent validity as acceptable based on level of significance $(p \leq 0.05)$, rather than the strength of the relationship (i.e., magnitude of the $r$-values). Accordingly, the strength of the relationship should be 
accounted for by future studies, as larger sample sizes are likely to generate associations at a statistically significant level, irrespective of the strength of the relationship. While the methodological quality of measurement error was rated as "good" to "excellent", the four PAQs corresponding to these ratings (i.e., YPAS, AAS [modified], PASE and VAPAQ) were classified as "indeterminate" for psychometric quality. This is because the included studies did not report minimal important change (MIC) with respect to smallest detectable change (SDC), or whether MIC ranged beyond the limits of agreement (LOA). According to Terwee, Roorda [44], SDC and MIC are essential parameters for reliability to allow better interpretation of change scores. Subsequently, more studies need to incorporate measurement error when examining reliability of PAQs and consider calculations of MIC and SDC and/or LOA for this psychometric property.

\section{Limitation}

The primary purpose of the current review was to examine the psychometric properties of interview-administered, Past-week and Usual-week PAQs in an adult population. Thus, investigating the psychometric properties of PAQs with recall time-frames beyond, or within, the 7-day period was beyond the scope of the study. In addition, the current review selectively included studies that examined the psychometric properties of PAQs that were published in an English-speaking country because cultural diversity appears to impact on the psychometric properties of PAQs (e.g., errors of translation between languages, interpretation difficulties). Furthermore, the current review specifically selected studies that were conducted in an adult population, given that PAQs for children and adolescents are developed according to their literacy level. Thus, comparing the psychometric qualities of PAQs between studies that were conducted in English-speaking and non-English speaking countries and between age groups (i.e., children, adolescents and adults) may expand our knowledge on the usability of PAQs across different population groups. Whilst we made every effort to exclude studies that included participants with diagnosed cognitive impairment, the majority of the included studies did not screen for cognitive impairment. Therefore, future studies should consider conducting cognitive assessments to ensure that cognitive conditions are not influencing the psychometric properties of PAQs, particularly in older adults. With respect to abstract screening, there were discrepancies between those who conducted the literature search and those who screened the abstracts, which may have introduced selective bias. However, the reviewers were rigorously trained prior to abstract screening to ensure transparency of the inclusion criteria, and any disagreement between reviewers were resolved by the primary author (KD). Finally, examining the responsiveness and cross-cultural validity of PAQs was beyond the scope of this review. Therefore, comparing the psychometric quality of these properties between different PAQ types may allow better understanding of the sensitivity to changes in PA level.

\section{Conclusion}

The current review demonstrated that the psychometric quality of the majority of reported psychometric properties exhibited "negative" ratings. In addition, minimal differences were identified in the psychometric quality between Usual-week and Past-week PAQs. These findings suggested that the psychometric qualities of commonly used interview-administered PAQs are weak irrespective of recall methods. Therefore, caution should be used when measuring PA level using the PAQs included in this review. According to the weighted mean of the $r$-values, test-retest reliability was stronger for Usual-week PAQs compared with Past-week PAQs, although the reverse was identified for convergent validity for direct measures of PA level. These results indicate that Usual-week PAQs may be more suitable when identifying PA levels, and its corresponding association with physical fitness, of a large population for epidemiological studies. Conversely, Past-week PAQs may allow better detection of changes in PA level following an intervention. Finally, the interview-administered PAQs exhibited stronger convergent validity than previously reported for self-administered PAQs [16]. Therefore, whilst interview-administered PAQs may be time-consuming and cumbersome, researchers may opt to utilise this method over self-administered PAQs to obtain greater accuracy in physical activity level. However, irrespective of the strength of correlations, it is important to note that the quality of the measurement properties were either not examined or were quite poor. Subsequently, future studies should investigate the psychometric properties using more robust methodologies based on the COSMIN to better understand the usability of current PAQs, or to develop new PAQs by addressing issues identified in this review.

\footnotetext{
Abbreviations

AAS: Active Australia Survey; CaMos: Canadian Multicentre Osteoporosis Study; CAQ-PAl: College Alumni Questionnaire - Physical Activity Index: COSMIN: Consensus-based Standards for the Selection of Health Measurement Instrument; ICC: Intra-class correlation coefficient; IPAQLF: International Physical Activity Questionnaire Long Form; IPAQSF: International Physical Activity Questionnaire Short Form; IPEQWA: Incidental and planned exercise questionnaire; LOA: Limits of agreement; MAQ: Modified activity questionnaire; MET: Metabolic equivalent of task; MIC: Minimal important change; NE: Not evaluated; NHS II: Nurses' Health Study version II; NR: Not reported; NZPAQ-LF: New Zealand Physical Activity Questionnaire Long Form; NZPAQ-SF: New Zealand Physical Activity Questionnaire Short Form; PA: Physical activity; PAAQ: Physical activity adult questionnaire; PAQ: Physical activity questionnaire; PAR: Physical activity recall questionnaire; PASE: Physical activity scale for the elderly; Phone-
} 
FITT: Phone fitness; PRISMA: Preferred reporting items for systematic reviews and meta-analyses; PWMAQ: Past week modified activity questionnaire: SCD: Smallest detectable change; VAPAQ: Veterans Physical Activity Questionnaire; WHO: World health organisation; YPAS: Yale Physical Activity Survey

\section{Acknowledgements}

The authors would like to thank Dr. Peter Fowler and Dr. Colette Thomas for their assistance with screening and compilation of full text articles.

\section{Funding}

The authors did not receive funding for this systematic review.

\section{Availability of data and materials}

All data are present in the tables and figures.

\section{Authors' contributions}

$\mathrm{KD}$ contributed to the concept, design of work, acquisition of analysis, interpretation of data and drafting of work and approved the submitted versions and agreed both to be personally accountable for the author's own contributions and to ensure that questions related to the accuracy or integrity of any part of the work, even ones in which the author was not personally involved, are appropriately investigated, resolved, and the resolution documented in the literature. RS contributed to the concept, design of work, interpretation of data and drafting and revision of work and approved the submitted versions and agreed both to be personally accountable for the author's own contributions and to ensure that questions related to the accuracy or integrity of any part of the work, even ones in which the author was not personally involved, are appropriately investigated, resolved, and the resolution documented in the literature. LP contributed to acquisition of data, analysis of data and drafting of work and approved the submitted versions and agreed both to be personally accountable for the author's own contributions and to ensure that questions related to the accuracy or integrity of any part of the work, even ones in which the author was not personally involved, are appropriately investigated, resolved, and the resolution documented in the literature. RC contributed to the concept, design of work, interpretation of data and drafting and revision of work and approved the submitted versions and agreed both to be personally accountable for the author's own contributions and to ensure that questions related to the accuracy or integrity of any part of the work, even ones in which the author was not personally involved, are appropriately investigated, resolved, and the resolution documented in the literature. All authors read and approved the final manuscript.

\section{Ethics approval and consent to participate}

Ethics approval was not applicable as this manuscript was a systematic review.

\section{Consent for publication}

Not applicable

\section{Competing interests}

The authors declare that they have no competing interests.

\section{Publisher's Note}

Springer Nature remains neutral with regard to jurisdictional claims in published maps and institutional affiliations.

\section{Author details}

${ }^{1}$ College of Healthcare Sciences, James Cook University, Townsville, Queensland, Australia. ${ }^{2}$ Department Special needs Education, University of Oslo, Oslo, Norway. ${ }^{3}$ School of Occupational Therapy, Social Work and Speech Pathology, Curtin University, Perth, Western Australia. ${ }^{4}$ Department of Otorhinolaryngology and Head and Neck Surgery, Leiden University Medical Center, Leiden, the Netherlands.
Received: 8 October 2018 Accepted: 14 February 2019

Published online: 01 March 2019

\section{References}

1. Rush E, Simmons D. Physical activity in children: prevention of obesity and type 2 diabetes. Med Sport Sci. 2014;60:113-21.

2. Pedersen BK, Saltin B. Exercise as medicine - evidence for prescribing exercise as therapy in 26 different chronic diseases. Scand J Med Sci Sports. 2015;25(Suppl 3):1-72

3. Doma K, Grant A, Morris J. The effects of balance training on balance performance and functional outcome measures following Total knee arthroplasty: a systematic review and meta-analysis. Sports Med. 2018; 48(10):2367-85.

4. Pierce DR, Doma K, Leicht AS. Acute effects of exercise mode on arterial stiffness and wave reflection in healthy young adults: a systematic review and meta-analysis. Front Physiol. 2018;9:73.

5. Poirier P, Giles TD, Bray GA, Hong Y, Stern JS, Pi-Sunyer FX, et al. Obesity and cardiovascular disease: pathophysiology, evaluation, and effect of weight loss: an update of the 1997 American Heart Association scientific statement on obesity and heart disease from the obesity Committee of the Council on nutrition, physical activity, and metabolism. Circulation. 2006:113(6):898-918.

6. Fussenich LM, Boddy LM, Green DJ, Graves LE, Foweather L, Dagger RM, et al. Physical activity guidelines and cardiovascular risk in children: a cross sectional analysis to determine whether 60 minutes is enough. BMC Public Health. 2016;16(1):67.

7. Oja P, Titze S. Physical activity recommendations for public health: development and policy context. EPMA J. 2011;2(3):253-9.

8. Babor TF, Sciamanna CN, Pronk NP. Assessing multiple risk behaviors in primary care. Screening issues and related concepts. Am J Prev Med. 2004;27(2 Suppl):42-53.

9. Lee PH, Macfarlane DJ, Lam TH, Stewart SM. Validity of the international physical activity questionnaire short form (IPAQ-SF): a systematic review. Int J Behav Nutr Phys Act. 2011;8:115.

10. Ainsworth BE, Caspersen CJ, Matthews CE, Masse LC, Baranowski T, Zhu W. Recommendations to improve the accuracy of estimates of physical activity derived from self report. J Phys Act Health. 2012;9(Suppl 1):S76-84.

11. Shephard RJ, Aoyagi Y. Seasonal variations in physical activity and implications for human health. Eur J Appl Physiol. 2009;107(3):251-71.

12. Merkx A, Ausems M, Bude L, de Vries R, Nieuwenhuijze MJ. Factors affecting perceived change in physical activity in pregnancy. Midwifery. 2017;51:16-23.

13. Terwee CB, Mokkink LB, Knol DL, Ostelo RW, Bouter LM, de Vet HC. Rating the methodological quality in systematic reviews of studies on measurement properties: a scoring system for the COSMIN checklist. Qual Life Res. 2012;21(4):651-7.

14. Schellingerhout JM, Verhagen AP, Heymans MW, Koes BW, de Vet HC, Terwee CB. Measurement properties of disease-specific questionnaires in patients with neck pain: a systematic review. Qual Life Res. 2012;21(4):659-70.

15. Terwee CB, Bot SD, de Boer MR, van der Windt DA, Knol DL, Dekker J, et al. Quality criteria were proposed for measurement properties of health status questionnaires. J Clin Epidemiol. 2007;60(1):34-42.

16. Doma K, Speyer R, Leicht AS, Cordier R. Comparison of psychometric properties between usual-week and past-week self-reported physical activity questionnaires: a systematic review. Int J Behav Nutr Phys Act. 2017;14(1):10.

17. Brown WJ, Burton NW, Marshall AL, Miller YD. Reliability and validity of a modified self-administered version of the active Australia physical activity survey in a sample of mid-age women. Aust N Z J Public Health. 2008;32(6):535-41.

18. Fjeldsoe BS, Winkler EA, Marshall AL, Eakin EG, Reeves MM. Active adults recall their physical activity differently to less active adults: test-retest reliability and validity of a physical activity survey. Health Promot J Austr. 2013;24(1):26-31.

19. Touvier M, Kesse-Guyot E, Mejean C, Pollet C, Malon A, Castetbon K, et al. Comparison between an interactive web-based self-administered $24 \mathrm{~h}$ dietary record and an interview by a dietitian for large-scale epidemiological studies. Br J Nutr. 2011;105(7):1055-64.

20. Bowling A. Mode of questionnaire administration can have serious effects on data quality. J Public Health (Oxf). 2005:27(3):281-91.

21. Okamoto K, Ohsuka K, Shiraishi T, Hukazawa E, Wakasugi S, Furuta K. Comparability of epidemiological information between self- and interviewer-administered questionnaires. J Clin Epidemiol. 2002:55(5):505-11. 
22. Schnall AH, Wolkin A, Nakata N. In: Horney JA, editor. Chapter 9 Methods: questionnaire development and interviewing techniques. London: Mica Haley; 2018

23. Forsen L, Loland NW, Vuillemin A, Chinapaw MJ, van Poppel MN, Mokkink $L B$, et al. Self-administered physical activity questionnaires for the elderly: a systematic review of measurement properties. Sports Med. 2010;40(7):601-23.

24. van Poppel MN, Chinapaw MJ, Mokkink LB, van Mechelen W, Terwee CB. Physical activity questionnaires for adults: a systematic review of measurement properties. Sports Med. 2010;40(7):565-600.

25. Liberati A, Altman DG, Tetzlaff J, Mulrow C, Gotzsche PC, loannidis JP, et al The PRISMA statement for reporting systematic reviews and meta-analyses of studies that evaluate healthcare interventions: explanation and elaboration. BMJ. 2009:339:b2700.

26. Cicchetti DV. Guidelines, criteria, and rules of thumb for evaluating normed and standardized assessment instruments in psychology. Psychol Assess. 1994;6(4):284-90.

27. Mokkink LB, Terwee CB, Patrick DL, Alonso J, Stratford PW, Knol DL, et al, The COSMIN checklist for assessing the methodological quality of studies on measurement properties of health status measurement instruments: an international Delphi study. Qual Life Res. 2010;19(4):539-49.

28. Aparicio-Ugarriza R, Mielgo-Ayuso J, Benito PJ, Pedrero-Chamizo R, Ara I, Gonzalez-Gross M, et al. Physical activity assessment in the general population; instrumental methods and new technologies. Nutr Hosp. 2015; 31(Suppl 3):219-26.

29. Cordier R, Speyer R, Chen YW, Wilkes-Gillan S, Brown T, Bourke-Taylor H, et al. Evaluating the psychometric quality of social skills measures: a systematic review. PLoS One. 2015;10(7):e0132299.

30. Cordier R, Chen YW, Speyer R, Totino R, Doma K, Leicht A, et al. Child-report measures of occupational performance: a systematic review. PLoS One. 2016;11(1):e0147751.

31. Cohen J. Statistical power analysis for the behavioral sciences. Hillsdale: Lawrence Erlbaum Associates; 1988.

32. Hakkinen A, Malkia E, Hakkinen K, Jappinen I, Laitinen L, Hannonen P. Effects of detraining subsequent to strength training on neuromuscular function in patients with inflammatory arthritis. Br J Rheumatol. 1997;36(10):1075-81.

33. Sarkin JA, Johnson SS, Prochaska JO, Prochaska JM. Applying the transtheoretical model to regular moderate exercise in an overweight population: validation of a stages of change measure. Prev Med. 2001:33(5):462-9.

34. de Vet HC, Terwee CB, Knol DL, Bouter LM. When to use agreement versus reliability measures. J Clin Epidemiol. 2006;59(10):1033-9.

35. Delbaere K, Hauer K, Lord SR. Evaluation of the incidental and planned activity questionnaire (IPEQ) for older people. Br J Sports Med. 2010;44(14):1029-34.

36. Chu AH, Ng SH, Koh D, Muller-Riemenschneider F. Reliability and validity of the self- and interviewer-administered versions of the global physical activity questionnaire (GPAQ). PLoS One. 2015;10(9):e0136944.

37. Vuillemin A, Oppert JM, Guillemin F, Essermeant L, Fontvieille AM, Galan P, et al. Self-administered questionnaire compared with interview to assess past-year physical activity. Med Sci Sports Exerc. 2000;32(6):1119-24.

38. Mujika I, Padilla S. Muscular characteristics of detraining in humans. Med Sci Sports Exerc. 2001;33(8):1297-303.

39. Pettee Gabriel K, McClain JJ, High RR, Schmid KK, Whitfield GP, Ainsworth BE. Patterns of accelerometer-derived estimates of inactivity in middle-age women. Med Sci Sports Exerc. 2012;44(1):104-10.

40. Rehn TA, Munkvik M, Lunde PK, Sjaastad I, Sejersted OM. Intrinsic skeletal muscle alterations in chronic heart failure patients: a disease-specific myopathy or a result of deconditioning? Heart Fail Rev. 2012;17(3):421-36.

41. Bickel CS, Cross JM, Bamman MM. Exercise dosing to retain resistance training adaptations in young and older adults. Med Sci Sports Exerc. 2011;43(7):1177-87.

42. Reimers AK, Mess F, Bucksch J, Jekauc D, Woll A. Systematic review on measurement properties of questionnaires assessing the neighbourhood environment in the context of youth physical activity behaviour. BMC Public Health. 2013;13:461.

43. Silsbury Z, Goldsmith R, Rushton A. Systematic review of the measurement properties of self-report physical activity questionnaires in healthy adult populations. BMJ Open. 2015;5(9):e008430.

44. Terwee CB, Roorda LD, Knol DL, De Boer MR, De Vet HC. Linking measurement error to minimal important change of patient-reported outcomes. J Clin Epidemiol. 2009;62(10):1062-7.

45. Nadalin V, Bentvelsen K, Kreiger N. Reliability of self-reports: data from the Canadian multi-Centre osteoporosis study (CaMos). Chronic Dis Canada. 2004;25(2):28-31.
46. Merom D, Delbaere K, Cumming R, Voukelatos A, Rissel C, Van Der Ploeg $\mathrm{HP}$, et al. Incidental and planned exercise questionnaire for seniors: validity and responsiveness. Med Sci Sports Exerc. 2014;46(5):947-54.

47. Pettee Gabriel K, McClain JJ, Lee CD, Swan PD, Alvar BA, Mitros MR, et al. Evaluation of physical activity measures used in middle-aged women. Med Sci Sports Exerc. 2009;41(7):1403-12.

48. Kriska AM, Knowler WC, LaPorte RE, Drash AL, Wing RR, Blair SN, et al. Development of questionnaire to examine relationship of physical activity and diabetes in Pima Indians. Diabetes Care. 1990;13(4):401-11.

49. Kriska AM, Edelstein SL, Hamman RF, Otto A, Bray GA, Mayer-Davis EJ, et al. Physical activity in individuals at risk for diabetes: diabetes prevention program. Med Sci Sports Exerc. 2006;38(5):826-32.

50. Schulz LO, Harper IT, Smith CJ, Kriska AM, Ravussin E. Energy intake and physical activity in Pima Indians: comparison with energy expenditure measured by doubly-labeled water. Obes Res. 1994;2(6):541-8.

51. Gill DP, Jones GR, Zou GY, Speechley M. The phone-FITT: a brief physical activity interview for older adults. J Aging Phys Act. 2008;16(3):292-315.

52. Colbert LH, Matthews CE, Havighurst TC, Kim K, Schoeller DA. Comparative validity of physical activity measures in older adults. Med Sci Sports Exerc. 2011;43(5):867-76

53. Dipietro L, Caspersen CJ, Ostfeld AM, Nadel ER. A survey for assessing physical activity among older adults. Med Sci Sports Exerc. 1993;25(5):628-42.

54. Gennuso KP, Matthews CE, Colbert LH. Reliability and validity of 2 self-report measures to assess sedentary behavior in older adults. J Phys Act Health. 2015;12(5):727-32.

55. Harada ND, Chiu V, King AC, Stewart AL. An evaluation of three selfreport physical activity instruments for older adults. Med Sci Sports Exerc. 2001;33(6):962-70.

56. Kolbe-Alexander TL, Lambert EV, Harkins JB, Ekelund U. Comparison of two methods of measuring physical activity in south African older adults. J Aging Phys Act. 2006;14(1):98-114.

57. Moore DS, Ellis R, Allen PD, Cherry KE, Monroe PA, O'Neil CE, et al. Construct validation of physical activity surveys in culturally diverse older adults: a comparison of four commonly used questionnaires. Res Q Exerc Sport. 2008;79(1):42-50.

58. Brown WJ, Trost SG, Bauman A, Mummery K, Owen N. Test-retest reliability of four physical activity measures used in population surveys. J Sci Med Sport. 2004:7(2):205-15.

59. Brown W, Bauman A, Chey T, Trost S, Mummery K. Comparison of surveys used to measure physical activity. Aust N Z J Public Health. 2004;28(2):128-34.

60. Creamer M, Bowles HR, von Hofe B, Pettee Gabriel K, Kohl HW 3rd, Bauman A. Utility of computer-assisted approaches for population surveillance of physical activity. J Phys Act Health. 2014;11(6):1111-9.

61. Mahabir S, Baer DJ, Giffen C, Clevidence BA, Campbell WS, Taylor PR, et al. Comparison of energy expenditure estimates from 4 physical activity questionnaires with doubly labeled water estimates in postmenopausal women. Am J Clin Nutr. 2006;84(1):230-6.

62. Rauh MJ, Hovell MF, Hofstetter CR, Sallis JF, Gleghorn A. Reliability and validity of self-reported physical activity in Latinos. Int J Epidemiol. 1992;21(5):966-71.

63. Washburn RA, Smith KW, Goldfield SR, McKinlay JB. Reliability and physiologic correlates of the Harvard alumni activity survey in a general population. J Clin Epidemiol. 1991;44(12):1319-26.

64. Masse LC, Fulton JE, Watson KB, Tortolero S, Kohl HW 3rd, Meyers MC, et al. Comparing the validity of 2 physical activity questionnaire formats in African-American and Hispanic women. J Phys Act Health. 2012;9(2):237-48.

65. Ahn GE, Chmiel JS, Dunlop DD, Helenowski IB, Semanik PA, Song J, et al. Self-reported and objectively measured physical activity in adults with systemic lupus erythematosus. Arthritis Care Res (Hoboken). 2015;67(5):701-7.

66. Garriguet D, Tremblay S, Colley RC. Comparison of physical activity adult questionnaire results with accelerometer data. Health Rep. 2015;26(7):11-7.

67. Ainsworth BE, Macera CA, Jones DA, Reis JP, Addy CL, Bowles HR, et al. Comparison of the 2001 BRFSS and the IPAQ physical activity questionnaires. Med Sci Sports Exerc. 2006;38(9):1584-92.

68. Moy KL, Scragg RK, McLean G, Carr H. The New Zealand physical activity questionnaires: validation by heart-rate monitoring in a multiethnic population. J Phys Act Health. 2008;5(Suppl 1):S45-61.

69. Dinger MK, Oman RF, Taylor EL, Vesely SK, Able J. Stability and convergent validity of the physical activity scale for the elderly (PASE). J Sports Med Phys Fitness. 2004;44(2):186-92. 
70. Johansen KL, Painter P, Kent-Braun JA, Ng AV, Carey S, Da Silva M, et al. Validation of questionnaires to estimate physical activity and functioning in end-stage renal disease. Kidney Int. 2001;59(3):1121-7.

71. Washburn RA, Smith KW, Jette AM, Janney CA. The physical activity scale for the elderly (PASE): development and evaluation. I Clin Epidemiol. 1993;46(2):153-62.

72. Pettee Gabriel K, McClain JJ, Schmid KK, Storti KL, Ainsworth BE. Reliability and convergent validity of the past-week modifiable activity questionnaire. Public Health Nutr. 2011;14(3):435-42.

73. Albanes D, Conway JM, Taylor PR, Moe PW, Judd J. Validation and comparison of eight physical activity questionnaires. Epidemiology (Cambridge, Mass). 1990;1 (1):65-71.

74. Blair SN, Haskell WL, Ho P, Paffenbarger RS Jr, Vranizan KM, Farquhar JW, et al. Assessment of habitual physical activity by a seven-day recall in a community survey and controlled experiments. Am J Epidemiol. 1985; 122(5):794-804.

75. Conway JM, Seale JL, Jacobs DR Jr, Irwin ML, Ainsworth BE. Comparison of energy expenditure estimates from doubly labeled water, a physical activity questionnaire, and physical activity records. Am J Clin Nutr. 2002;75(3):519-25.

76. Garfield BE, Canavan JL, Smith CJ, Ingram KA, Fowler RP, Clark AL, et al. Stanford seven-day physical activity recall questionnaire in COPD. Eur Respir J. 2012:40(2):356-62

77. Gross LD, Sallis JF, Buono MJ, Roby JJ, Nelson JA. Reliability of interviewers using the seven-day physical activity recall. Res Q Exerc Sport. 1990;61(4):321-5.

78. Irwin ML, Ainsworth $\mathrm{BE}$, Conway JM. Estimation of energy expenditure from physical activity measures: determinants of accuracy. Obes Res. 2001:9(9):517-25.

79. Sallis JF, Haskell WL, Wood PD, Fortmann SP, Rogers T, Blair SN, et al. Physical activity assessment methodology in the Five-City project. Am J Epidemiol. 1985;121(1):91-106.

80. Taylor CB, Coffey T, Berra K, laffaldano R, Casey K, Haskell WL. Seven-day activity and self-report compared to a direct measure of physical activity. Am J Epidemiol. 1984;120(6):818-24.

81. Washburn RA, Jacobsen DJ, Sonko BJ, Hill JO, Donnelly JE. The validity of the Stanford seven-day physical activity recall in young adults. Med Sci Sports Exerc. 2003:35(8):1374-80.

82. Williams E, Klesges RC, Hanson CL, Eck LH. A prospective study of the reliability and convergent validity of three physical activity measures in a field research trial. J Clin Epidemiol. 1989;42(12):1161-70.

83. Betz HH, Myers J, Jaffe A, Smith K, Dalman R. Reproducibility of the veterans physical activity questionnaire in an elderly population. J Phys Act Health. 2015;12(3):376-81.

Ready to submit your research? Choose BMC and benefit from:

- fast, convenient online submission

- thorough peer review by experienced researchers in your field

- rapid publication on acceptance

- support for research data, including large and complex data types

- gold Open Access which fosters wider collaboration and increased citations

- maximum visibility for your research: over $100 \mathrm{M}$ website views per year

At $\mathrm{BMC}$, research is always in progress.

Learn more biomedcentral.com/submissions 